\title{
Matchings and Partial Patterns
}

\author{
Vít Jelínek* \\ Fakultät für Mathematik, Universität Wien \\ Garnisongasse 3, 1090 Vienna, Austria \\ jelinek@kam.mff.cuni.cz \\ Toufik Mansour \\ Department of Mathematics, Haifa University \\ 31905 Haifa, Israel \\ toufik@math.haifa.ac.il
}

Submitted: May 21, 2010; Accepted: Nov 14, 2010; Published: Nov 26, 2010

Mathematics Subject Classification: Primary: 05A18; Secondary: 05A05, 05A15

\begin{abstract}
A matching of size $2 n$ is a partition of the set $[2 n]=\{1,2, \ldots, 2 n\}$ into $n$ disjoint pairs. A matching may be identified with a canonical sequence, which is a sequence of integers in which each integer $i \in[n]$ occurs exactly twice, and the first occurrence of $i$ precedes the first occurrence of $i+1$. A partial pattern with $k$ symbols is a sequence of integers from the set $[k]$, in which each $i \in[k]$ appears at least once and at most twice, and the first occurrence of $i$ always precedes the first occurrence of $i+1$.

Given a partial pattern $\sigma$ and a matching $\mu$, we say that $\mu$ avoids $\sigma$ if the canonical sequence of $\mu$ has no subsequence order-isomorphic to $\sigma$. Two partial patterns $\tau$ and $\sigma$ are equivalent if there is a size-preserving bijection between $\tau$ avoiding and $\sigma$-avoiding matchings. In this paper, we describe several families of equivalent pairs of patterns, most of them involving infinitely many equivalent pairs. We verify by computer enumeration that these families contain all the equivalences among patterns of length at most six. Many of our arguments exploit a close connection between partial patterns and fillings of diagrams.
\end{abstract}

\section{Introduction}

A matching on a vertex set $[2 n]=\{1,2, \ldots, 2 n\}$ is a partition of $[2 n]$ into disjoint blocks of size two, or equivalently, a graph on $[2 n]$ in which every vertex has degree one. The

\footnotetext{
*Supported by grant no. 090038011 from the Icelandic Research Fund and by grant Z130-N13 from the Austrian Science Foundation (FWF).
} 
number of edges of a matching $\mu$ will be referred to as the order of $\mu$. The set of matchings on $[2 n]$ is denoted by $\mathcal{M}_{n}$.

In this paper, we identify a matching $\mu \in \mathcal{M}_{n}$ with a sequence of $2 n$ integers from the set $[n]$ such that each number $i \in[n]$ appears exactly twice, and the first occurrence of $i$ precedes the first occurrence of $j$ whenever $i<j$. Such a representation is the canonical sequence [9] of $\mu$. In this representation, vertices of a matching correspond to elements of the canonical sequence, and two vertices are connected by an edge if and only if the corresponding elements of the canonical sequence are equal. For example, the matching in Figure 1 is represented by the canonical sequence 123321.

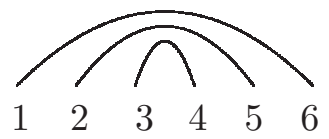

Figure 1: The matching 123321.

A partial pattern (or just pattern) of length $m$ with $k$ symbols is a sequence $\sigma=$ $\sigma_{1} \sigma_{2} \cdots \sigma_{m}$ of integers from the set $[k]$, with the property that each number $i \in[k]$ appears at least once and at most twice in $\sigma$, and the first occurrence of a number $i \in[k]$ precedes the first occurrence of $j \in[k]$ whenever $i<j$. Naturally, a matching is a special case of a partial pattern.

Let $s=s_{1} s_{2} \cdots s_{m}$ and $t=t_{1} t_{2} \cdots t_{m}$ be two sequences of integers. We say that $s$ and $t$ are order-isomorphic if for any $i, j \in[m]$, the inequality $s_{i}<s_{j}$ holds if and only if $t_{i}<t_{j}$ holds.

We say that a matching $\mu$ contains a pattern $\sigma$, if $\mu$ has a subsequence that is orderisomorphic to $\sigma$. If $\mu$ does not contain $\sigma$, we say that $\mu$ avoids $\sigma$ or $\mu$ is $\sigma$-avoiding. We let $\mathcal{M}_{n}(\sigma)$ denote the set of $\sigma$-avoiding matchings on $[2 n]$, and we let $\mathfrak{m}_{n}(\sigma)$ denote the cardinality of $\mathcal{M}_{n}(\sigma)$. We say that two patterns $\sigma$ and $\tau$ are equivalent, denoted by $\sigma \sim \tau$, if $\mathfrak{m}_{n}(\sigma)=\mathfrak{m}_{n}(\tau)$ for every $n \in \mathbb{N}$.

The goal of this paper is to find families of equivalent patterns. Rather than constructing single-purpose bijections between fixed pairs of pattern avoiding classes, we prefer to focus on results involving infinite families of equivalent pairs of patterns.

In Section 2, we give a summary of previous results on equivalences between patterns, including results have been obtained in the more general context of pattern avoidance of set partitions. In Section 3, we prove several results that show how shorter patterns can be combined into longer ones in a manner that preserves equivalence.

In Section 4, we deal with the relationship between pattern avoiding matchings and fillings of diagrams. We first show that the concept of shape-Wilf equivalence, which has been introduced in the context of pattern avoiding permutations, has direct implications for pattern avoiding matchings. Specifically, we show that any pair of shape-Wilf equivalent matrices gives rise to an infinite family of equivalent pairs of partial patterns. Next, we provide a more complicated construction that establishes a correspondence between fillings of stack diagrams and pattern avoidance in matchings. This correspondence allows us to use results of Rubey [11] on diagonal-avoiding stack fillings to obtain new families 
of equivalent patterns. We also prove a new result about pattern avoiding stack fillings which can be used for our purposes as well.

In Section 5, we give a different argument, based on the concept of 'hybrid' matchings, which allows us to obtain more examples of equivalent patterns. Finally, in Section 6, we summarize the implications of our results for patterns of length up to seven, and we state several results on the enumeration of specific pattern avoiding classes. We verified by computer enumeration that our results explain all the equivalences among patterns of length at most six, while there are several patterns of length seven that seem to be equivalent but are not covered by our results. We list these open cases at the end of the paper, as Conjecture 6.2.

Let us fix several useful notational conventions that we will apply throughout this paper. If $s=s_{1} s_{2} \cdots s_{n}$ is a sequence of integers and $k$ is an integer, we let $s+k$ denote the sequence of integers $\left(s_{1}+k\right)\left(s_{2}+k\right) \cdots\left(s_{n}+k\right)$. If $s=s_{1} \ldots s_{n}$ and $t=t_{1} \cdots t_{m}$ are two sequences, then st is their concatenation $s_{1} \cdots s_{n} t_{1} \cdots t_{m}$. In our arguments, it often crucial to maintain the distinction between sequences of integers that represent matchings, partitions and partial patterns, as opposed to arbitrary unrestricted sequences of integers. To stress the distinction, we adopt the convention of using lowercase Greek letters for matchings, partitions and patterns, while using lowercase Latin letters for arbitrary sequences. A sequence over the alphabet $[k]$ is any sequence of integers whose elements all belong to $[k]=\{1, \ldots, k\}$. Note that this does not imply that each element of $[k]$ must appear in the sequence. When referring to sequences, the notation $i^{k}$ denotes the constant sequence $i, i, \ldots, i$ of length $k$.

\section{Previous work}

Several natural classes of matchings can be characterized in terms of pattern avoidance. For instance, the classes $\mathcal{M}_{n}(1212)$ and $\mathcal{M}_{n}(1221)$ are known, respectively, as non-crossing and non-nesting matchings. These matchings are enumerated by the Catalan numbers $C_{n}=\frac{1}{n+1}\left(\begin{array}{c}2 n \\ n\end{array}\right)$ (see, e.g., [14]).

More generally, for an integer $k$, the matchings that avoid the pattern $12 \cdots k 12 \cdots k$ are known as $k$-noncrossing matchings, and matchings avoiding the pattern $12 \cdots k k(k-$ 1) $\cdots 21$ are known as $k$-nonnesting matchings. Chen et al. [2] have found a bijection between these two classes of matchings, thus showing that the patterns $12 \cdots k 12 \cdots k$ and $12 \cdots k k(k-1) \cdots 21$ are equivalent for any $k$. This result can be generalized to the broader setting of set partitions (see Fact 2.1).

Another class of pattern avoiding matchings that has been previously studied is the class $\mathcal{M}_{n}(123 \cdots k 1)$, where $k \geqslant 2$ is an integer. Chen, Xin and Zhang [5] have shown that the generating function

$$
f_{k}(x)=\sum_{n \geqslant 0} \mathfrak{m}_{n}(12 \cdots k 1) x^{n}
$$


is rational for any $k$, and provided the following explicit formulas:

$$
\begin{aligned}
& f_{3}(x)=\frac{1-x}{1-2 x-x^{2}} \\
& f_{4}(x)=\frac{1-2 x-2 x^{2}-x^{3}}{1-3 x-2 x^{2}-5 x^{3}-x^{4}} .
\end{aligned}
$$

Apart from these general results, various authors have studied classes of matchings avoiding a particular fixed pattern of small size. Jelínek, Li, Mansour and Yan [8] have shown that the matchings avoiding 1123 correspond bijectively to a certain class of lattice paths, and used this fact to obtain the formula

$$
\mathfrak{m}_{n}(1123)=\frac{3}{2 n+1}\left(\begin{array}{c}
2 n+1 \\
n-1
\end{array}\right) .
$$

Chen, Mansour and Yan [4] have shown that

$$
\mathfrak{m}_{n}(12312)=\frac{1}{2 n+1}\left(\begin{array}{c}
3 n \\
n
\end{array}\right)
$$

and they proved that the patterns 12312, 12321, 12231, 12213, 12132 and 12123 are all equivalent. This too can be generalized to set partitions, see Fact 2.6.

\subsection{The relationship between matchings and set partitions}

Since a matching is a special case of a set partition, many results on pattern avoiding set partitions are relevant to the study of pattern avoiding matchings. A set partition of the set $[n]$ is a set of disjoint nonempty sets $\left\{B_{1}, \ldots, B_{k}\right\}$, called blocks, whose union is $[n]$. We assume that the blocks are numbered in such a way that the smallest element of a block $B_{i}$ is smaller than the smallest element of $B_{j}$ whenever $i<j$. A set partition may be identified with a canonical sequence $\pi=\pi_{1} \cdots \pi_{n}$, defined by putting $\pi_{j}=i$ when $j \in B_{i}$. In the special case when all the blocks have size two, this definition of canonical sequence coincides with the definition we gave in the introduction.

We say that a partition $\pi$ contains a partition $\rho$ if the canonical sequence of $\pi$ has a subsequence order-isomorphic to the canonical sequence of $\rho$. Otherwise we say that $\pi$ avoids $\rho$. This concept of pattern avoidance has been introduced by Sagan [12] and later studied by the authors of this paper [7].

Let $P_{n}(\tau)$ be the set of all the partitions of $[n]$ that avoid the pattern $\tau$. Furthermore, if $a_{1}, \ldots, a_{m}$ is a sequence of numbers whose sum is $n$, let $P\left(\tau ; a_{1}, \ldots, a_{m}\right)$ be the set of all the partitions from $P_{n}(\tau)$ that have $m$ blocks and their $i$-th block has size $a_{i}$. The cardinality of $P_{n}(\tau)$ and $P\left(\tau ; a_{1}, \ldots, a_{m}\right)$ will be denoted by $p_{n}(\tau)$ and $p\left(\tau ; a_{1}, \ldots, a_{m}\right)$.

We say that two partitions $\tau$ and $\sigma$ are partition-equivalent, if $p_{n}(\tau)=p_{n}(\sigma)$ for each $n$. We say that the two partitions are strongly partition-equivalent, if $p\left(\tau ; a_{1}, \ldots, a_{m}\right)=$ $p\left(\sigma ; a_{1}, \ldots, a_{m}\right)$ for every sequence of natural numbers $a_{1}, \ldots, a_{m}$.

A set partition whose blocks all have size 1 or 2 is a partial matching. In particular, a pattern is a partial matching. Two patterns $\sigma$ and $\tau$ are partial-matching 
equivalent if $p\left(\sigma ; a_{1}, \ldots, a_{m}\right)=p\left(\tau ; a_{1}, \ldots, a_{m}\right)$ for each sequence $a_{1}, \ldots, a_{m}$ with $a_{i} \in$ $\{1,2\}$. Clearly, strongly partition-equivalent patterns are partial-matching equivalent, and partial-matching equivalent patterns are equivalent. There exist pairs of patterns (e.g., 123134 and 123413) that are partial-matching equivalent but not partition-equivalent. There are also pairs of patterns, like 1231 and 1232, which are partition-equivalent but not equivalent.

In [7], several classes of partition-equivalent patterns are presented. Although the paper does not deal with strong partition equivalence explicitly, some of the proofs immediately yield strong partition-equivalence of the corresponding patterns. In particular, from [7] we may obtain the following results (the references in brackets point to the corresponding statements in [7]).

Fact 2.1 (Lemma 9, Theorem 18, Corollary 18). For every $k$ and every partition $\tau$ the two partitions $12 \cdots k(\tau+k) 12 \cdots k$ and $12 \cdots k(\tau+k) k(k-1) \cdots 1$ are strongly partitionequivalent.

Fact 2.2 (Lemma 11, Corollary 21). For every $k$ and every partition $\tau$ the two partitions $12 \cdots k 12 \cdots k(\tau+k)$ and $12 \cdots k k(k-1) \cdots 1(\tau+k)$ are strongly partition-equivalent.

Fact 2.3 (Corollary 40). For every $p, q \geqslant 0$, for every $k \geqslant 0$, and for every partition $\tau, 12 \cdots k(\tau+k) 2^{p} 12^{q}$ is strongly partition-equivalent to $12 \cdots k(\tau+k) 2^{p+q} 1$, and $12 \cdots k 2^{p} 12^{q}(\tau+k)$ is strongly partition-equivalent to $12 \cdots k 2^{p+q} 1(\tau+k)$.

Fact 2.4 (Theorem 41). For every partition $\tau$ with $m \geqslant 0$ blocks, for every $p \geqslant 1$ and $q \geqslant 0$, the partitions $\tau(m+1)^{p}(m+2)(m+1)^{q}$ and $\tau(m+1)^{p+q}(m+2)$ are strongly partition-equivalent.

Fact 2.5 (Theorem 42). For every sequence $s$ over the alphabet $[m]$, for every $p \geqslant 1$ and $q \geqslant 0$, the partitions $12 \cdots m(m+1)^{p}(m+2)(m+1)^{q} s$ and $12 \cdots m(m+1)^{p+q}(m+2) s$ are strongly partition-equivalent.

Fact 2.6 (Theorem 48). For every $k$, all the partitions of length $k$ that start with 12 and that contain two occurrences of the symbol 1, one occurrence of the symbol 3, and all their remaining symbols are equal to 2, are mutually strongly partition-equivalent.

Fact 2.7 (Theorem 54). For every $p, q \geqslant 0$, the following pairs of partitions are strongly partition-equivalent:

- $1232^{p} 142^{q}$ and $12312^{p} 42^{q}$

- $1232^{p} 412^{q}$ and $1232^{p} 42^{q} 1$

- $123^{p+1} 413^{q}$ and $12343^{p} 13^{q}$

- $123^{p+1} 143^{q}$ and $123^{p+1} 13^{q} 4$ 


\section{Longer patterns from short ones}

Before we deal with non-trivial results, we first state, without proof, three easy observations.

Observation 3.1. A matching avoids the pattern $123 \cdots k$ if and only if it has fewer than $k$ edges. The same is true for the pattern $12 \cdots k k$. Consequently, $\mathfrak{m}_{n}(12 \cdots k)=$ $\mathfrak{m}_{n}(12 \cdots k k)=(2 n-1) ! ! \delta_{n<k}$, where $\delta_{n<k}$ is equal to 1 if $n<k$ and 0 otherwise, while $(2 n-1) ! !=1 \cdot 3 \cdot 5 \cdot \cdots \cdot(2 n-1)$.

Observation 3.2. For any $n$, the only matching of order $n$ avoiding the pattern 112 is the matching $12 \cdots n n(n-1) \cdots 1$, and the only matching of order $n$ avoiding the pattern 121 is the matching $112233 \cdots n n$.

Observation 3.3. A matching $\mu$ of order $n$ avoids the pattern 1122 if and only if $\mu$ has the form $12 \cdots n s_{1} s_{2} \cdots s_{n}$, where $s_{1} \cdots s_{n}$ is a permutation of $[n]$. Therefore, $\mathfrak{m}_{n}(1122)=n$ ! for every $n$.

In the rest of this section, we present several results showing how from a given pair of equivalent patterns we can construct new equivalent pairs of longer patterns.

Lemma 3.4. For any pattern $\tau$, we have $\mathfrak{m}_{n}(1(\tau+1))=(2 n-1) \mathfrak{m}_{n-1}(\tau)$. Consequently, $\sigma \sim \tau$ implies $1(\sigma+1) \sim 1(\tau+1)$.

Proof. Let $\mu$ be a matching of order $n$, and let $\mu^{\prime}$ be a matching of order $n-1$ obtained by removing from $\mu$ the edge incident to the leftmost vertex. Notice that $\mu$ avoids $1(\tau+1)$ if and only if $\mu^{\prime}$ avoids $\tau$. Moreover, any $\tau$-avoiding matching $\mu^{\prime}$ of order $n-1$ can be extended into $2 n-1$ different $1(\tau+1)$-avoiding matchings of order $n$ by inserting into $\mu^{\prime}$ a new edge adjacent to a new leftmost vertex. This shows that $\mathfrak{m}_{n}(1(\tau+1))=$ $(2 n-1) \mathfrak{m}_{n-1}(\tau)$. This proves the first claim of the lemma. The second claim follows from the first.

Observations 3.1 and 3.2 together with Lemma 3.4 are sufficient to enumerate the matchings avoiding a pattern of length 3 .

Table 1: Values of $\mathfrak{m}_{n}(\tau)$, where $\tau$ is a pattern of length 3 .

\begin{tabular}{l|lllllll|l}
$\tau$ & $n=1$ & $n=2$ & $n=3$ & $n=4$ & $n=5$ & $n=6$ & $n=7$ & Reference \\
\hline 122 & 1 & 0 & 0 & 0 & 0 & 0 & 0 & Observation 3.1 \\
\hline 123 & 1 & 3 & 0 & 0 & 0 & 0 & 0 & Observation 3.1 \\
\hline 112,121 & 1 & 1 & 1 & 1 & 1 & 1 & 1 & Fact 2.4
\end{tabular}

Lemma 3.5. If $\tau$ is a pattern that contains two occurrences of the letter 1 , then the number of matchings on $[2 n]$ that avoid $11(\tau+1)$ is given by the formula

$$
\mathfrak{m}_{n}(11(\tau+1))=\sum_{\ell=1}^{n} \ell !\left(\begin{array}{c}
2 n-\ell-1 \\
\ell-1
\end{array}\right) \mathfrak{m}_{n-\ell}(\tau) .
$$


Proof. Let $\mu$ be an arbitrary matching on $2 n$ vertices. In the matching $\mu$, an edge $i$ connects a pair of vertices $l_{i}<r_{i}$, called left vertex and right vertex of $i$, respectively. We assume that the edges are numbered in such a way that $l_{i}<l_{i+1}$ for each $i<n$.

Let $x$ be the edge that is incident to the leftmost right vertex of $\mu$. In other words, $x$ is such that $r_{x}<r_{y}$ for every $y \neq x$. We say that an edge $i$ of $\mu$ is leftist if $l_{i}<r_{x}$ and an edge is rightist otherwise. Note that every leftist edge $i$ satisfies $l_{i}<r_{x} \leqslant r_{i}$. In particular, each two leftist edges either nest or cross.

Let $\tau$ be a pattern with two occurrences of the letter 1 . We claim that a matching $\mu$ avoids $11(\tau+1)$ if and only if the submatching of $\mu$ induced by the rightist edges avoids $\tau$.

To see this, note that if the rightist edges of $\mu$ contain the pattern $\tau$, then the rightist edges together with the edge $x$ contain the pattern $11(\tau+1)$. To prove the converse, assume that $\mu$ is a matching that contains a $k$-tuple of edges $e_{1}<e_{2}<\cdots<e_{k}$ that induce the pattern $11(\tau+1)$. Since $\tau$ has two occurrences of the symbol 1 , we know that both vertices incident to $e_{2}$ appear to the right of the two vertices incident to $e_{1}$. In particular, $e_{2}$ is a rightist edge. It follows that all the $k-1$ edges $e_{2}, e_{3}, \ldots, e_{k}$ are rightist, and these $k-1$ rightist edges contain the pattern $\tau$. This proves the claim from the previous paragraph.

To see that the claim implies the formula in the lemma, it suffices to observe that in $\mathcal{M}_{n}(11(\tau+1))$ there are exactly $\ell !\left(\begin{array}{c}2 n-\ell-1 \\ \ell-1\end{array}\right) \mathfrak{m}_{n-\ell}(\tau)$ matchings with $\ell$ leftist edges. Indeed, the factor $\ell$ ! counts the possible mutual positions of the leftist edges, the factor $\mathfrak{m}_{n-\ell}(\tau)$ counts the possible mutual positions of the rightist edges, and $\left(\begin{array}{c}2 n-\ell-1 \\ \ell-1\end{array}\right)$ is the number of ways to insert the right vertices of the $\ell-1$ leftist edges different from $x$ among the $2(n-\ell)$ vertices incident with the rightist edges. The lemma follows.

The following claim is a direct consequence of the previous lemma.

Corollary 3.6. If $\sigma$ and $\tau$ are two equivalent patterns that both have two occurrences of the symbol 1 , then $11(\sigma+1)$ and $11(\tau+1)$ are equivalent as well.

In the previous corollary, the assumption that both $\sigma$ and $\tau$ have two occurrences of the symbol 1 cannot be omitted. For instance, the two patterns 12 and 122 are equivalent (as shown by Observation 3.1), but the patterns 1123 and 11233 are not.

The next lemma is a generalization of Corollary 3.6.

Lemma 3.7. Let $\sigma$ and $\tau$ be two equivalent patterns that both have two occurrences of the symbol 1. Let $\rho$ be a pattern with $k$ distinct letters. Then the two patterns $\rho(\sigma+k)$ and $\rho(\tau+k)$ are also equivalent.

Proof. Let $\sigma^{\prime}$ and $\tau^{\prime}$ denote the patterns $\rho(\sigma+k)$ and $\rho(\tau+k)$, respectively. We will describe a procedure $g$ that bijectively transforms a $\sigma^{\prime}$-avoiding matching into a $\tau^{\prime}$-avoiding matching of the same length. Let $\mu \in \mathcal{M}_{n}$ be a matching, represented by its canonical sequence $\mu_{1} \mu_{2} \cdots \mu_{2 n}$.

Let $q=q(\mu)$ be the smallest integer such that the prefix $\mu_{1} \mu_{2} \cdots \mu_{q}$ of $\mu$ contains $\rho$. If $\mu$ avoids $\rho$, we define $q=2 n$. Let us also define $r=r(\mu)=\max \left\{\mu_{1}, \mu_{2}, \ldots, \mu_{q}\right\}$. Notice that each of the integers $1,2, \ldots, r$ must appear at least once in $\mu_{1}, \ldots, \mu_{q}$. Let $\mu_{>r}$ be 
the subsequence of $\mu$ formed by all the numbers in $\mu$ that are greater than $r$. Note that $\mu_{>r}$ is a sequence over the alphabet $\{r+1, r+2, \ldots, n\}$ in which each symbol appears exactly twice. Furthermore, $\mu_{>r}-r$ is a canonical sequence representing a matching with $n-r$ edges. Note also that all the elements of $\mu_{>r}$ are to the right of $\mu_{q}$.

We claim that $\mu$ contains $\sigma^{\prime}$ if and only if $\mu_{>r}$ contains $\sigma$. It is clear that if $\mu_{>r}$ contains $\sigma$, then $\mu$ contains $\sigma^{\prime}$. To prove the converse, assume that $\mu$ contains $\sigma^{\prime}$. Let $\ell$ be the length of $\sigma^{\prime}$, and let $s=s_{1} s_{2} \cdots s_{\ell}$ be the subsequence of $\mu$ that is order-isomorphic to $\sigma^{\prime}$. Since the pattern $\sigma$ has two occurrences of the symbol 1, the pattern $\sigma^{\prime}$ has two occurrences of $(k+1)$. Let $s_{i}$ and $s_{j}$ be the two elements of $s$ that correspond to the two occurrences of $(k+1)$ in $\sigma^{\prime}$, with $i<j$. This means that the sequence $s_{1}, s_{2}, \ldots, s_{i-1}$ is order-isomorphic to $\rho$, while $s_{i}, s_{i+1}, \ldots, s_{\ell}$ is order-isomorphic to $\sigma$. In particular, all the elements $s_{i}, \ldots, s_{\ell}$ appear strictly to the right of $\mu_{q}$ in $\mu$. Since each number from the set $\{1, \ldots, r\}$ appears at least once among $\mu_{1}, \ldots, \mu_{q}$, and since $s_{i}$ and $s_{j}$ both appear to the right of $\mu_{q}$, we conclude that $s_{i}>r$. Since $s_{i}$ is the minimum of the sequence $s_{i}, s_{i+1}, \ldots, s_{\ell}$, we see that all the elements of this sequence belong to $\mu_{>r}$, hence $\mu_{>r}$ contains $\sigma$, as claimed. By the same argument, $\mu$ contains $\tau^{\prime}$ if and only if $\mu_{>r}$ contains $\tau$.

We now describe the bijection between $\sigma^{\prime}$-avoiding and $\tau^{\prime}$-avoiding matchings. Let $\mu$ be a $\sigma^{\prime}$-avoiding matching that contains $\rho$. Let $q, r$ and $\mu_{>r}$ be as above. We know $\mu_{>r}-r$ is a canonical sequence representing a matching $\mu_{\sigma}$ that avoids $\sigma$. Since $\sigma$ and $\tau$ are equivalent, there is a function $f$ that maps $\sigma$-avoiding matchings bijectively to $\tau$-avoiding matchings of the same length. Define $\mu_{\tau}=f\left(\mu_{\sigma}\right)$. Let $\mu_{>r}^{\prime}$ be the sequence $\mu_{\tau}+r$. Let $\mu^{\prime}$ be the matching obtained from $\mu$ by replacing each symbol in the subsequence $\mu_{>r}$ with the corresponding symbol from $\mu_{>r}^{\prime}$. Note that the two matchings $\mu$ and $\mu^{\prime}$ have the same prefix of length $q$. In particular, $q(\mu)=q\left(\mu^{\prime}\right)$ and $r(\mu)=r\left(\mu^{\prime}\right)$. It is then clear that the mapping $\mu \mapsto \mu^{\prime}$ is the required bijection.

For a matching $\mu$ on the set $[2 n]$, we let $\bar{\mu}$ denote the reversal of $\mu$, i.e., the matching $\bar{\mu}$ contains the edge $i j$ if and only if $\mu$ contains the edge $(n-i+1)(n-j+1)$. For instance, the reversal of 112323 is 121233 .

Observation 3.8. If $\mu$ and $\nu$ are matchings, then $\mu$ contains $\nu$ if and only if $\bar{\mu}$ contains $\bar{\nu}$. Consequently, a matching $\mu$ is equivalent to its reversal $\bar{\mu}$.

Lemma 3.9. If $\tau$ is a matching on the set [2k-2], then the pattern $11(\tau+1)$ is equivalent to the pattern $\tau k k$.

Proof. It suffices to notice that $\tau k k=\overline{11 \overline{(\tau+1)}}$, and use Observation 3.8 and Corollary 3.6.

Combining Lemma 3.9 with Lemma 3.5, we observe that if $\tau$ and $\sigma$ are two equivalent matchings with $k-1$ edges, then $\tau k k$ and $\sigma k k$ are equivalent matchings with $k$ edges.

The next lemma extends this observation to more general cases. Its proof is based on similar ideas as the proof of Lemma 3.7.

Lemma 3.10. Let $\sigma$ and $\tau$ be two partial-matching equivalent patterns over the alphabet $[k]$, both of them containing each symbol from $[k]$ at least once. Let $\rho$ be a pattern that 
has two occurrences of the symbol 1. Then the two patterns $\sigma(\rho+k)$ and $\tau(\rho+k)$ are equivalent.

Proof. Let us write $\sigma^{\prime}=\sigma(\rho+k)$ and $\tau^{\prime}=\tau(\rho+k)$. Let $\mu=\mu_{1} \mu_{2} \cdots \mu_{2 n}$ be a matching. Let $q=q(\mu)$ be the largest integer such that the suffix $\mu_{q}, \mu_{q+1}, \ldots, \mu_{2 n}$ of $\mu$ contains $\rho$. If $\mu$ avoids $\rho$, we define $q=q(\mu)=1$. Define $r=r(\mu)=\mu_{q}$. Since $\rho$ has two occurrences of 1 , there must be two occurrences of $r$ in $\mu_{q}, \mu_{q+1}, \ldots, \mu_{2 n}$.

Let $\mu_{-}$denote the sequence $\mu_{1}, \mu_{2}, \ldots, \mu_{q-1}$. Notice that $\mu_{-}$is a partial matching over the alphabet $[r-1]$. It is easy to observe that $\mu$ contains $\sigma^{\prime}$ if and only if $\mu_{-}$contains $\sigma$, and $\mu$ contains $\tau^{\prime}$ if and only if $\mu_{-}$contains $\tau$. We now define the bijection between $\sigma^{\prime}$-avoiding and $\tau^{\prime}$-avoiding matchings. Since $\sigma$ and $\tau$ are assumed to be partial-matching equivalent, there is a bijection $f$ between $\sigma$-avoiding and $\tau$-avoiding partial matchings that preserves the sizes of the blocks. Let $\mu$ be a $\sigma^{\prime}$-avoiding matching. Then $\mu_{-}$is a $\sigma$-avoiding partial matching. Define $\mu_{-}^{\prime}=f\left(\mu_{-}\right)$. Let $\mu^{\prime}$ be the matching obtained from $\mu$ by replacing the prefix $\mu_{-}$with the prefix $\mu_{-}^{\prime}$. It is routine to check that the mapping $\mu \mapsto \mu^{\prime}$ is the required bijection.

In Lemma 3.10, unlike in Lemma 3.7, it is not enough to assume that $\sigma$ and $\tau$ are equivalent. For instance, the patterns 12 and 122 are equivalent, while the patterns 1233 and 12233 are not. The assumption that $\rho$ has two occurrences of the symbol 1 cannot be omitted either, since 121 and 112 are partial-matching equivalent, while 1213 and 1123 are not equivalent.

\section{Partial matchings and fillings of diagrams}

There is a very close relationship between canonical sequences and 01-fillings of diagrams. In this subsection, we will introduce the relevant terminology, and we will show how results on partial matchings can be seen as a consequence of results on pattern avoiding diagram fillings.

We will use the term diagram to refer to any finite set of the cells of the two-dimensional square grid. To fill a diagram means to assign a value of 0 or 1 to each cell.

We will number the rows of diagrams from bottom to top, so the "first row" of a diagram is its bottom row, and we will number the columns from left to right. We will apply the same convention to matrices and to fillings. We always assume that each row and each column of a diagram is nonempty. Thus, for example, when we refer to a diagram with $r$ rows, it is assumed that each of the $r$ rows contains at least one cell of the diagram. Note that there is a (unique) empty diagram with no rows and no columns.

A diagram $\Delta$ is row-convex, if it has the property that for any two of its cells $c_{1}$ and $c_{2}$ belonging to the same row, all the cells between $c_{1}$ and $c_{2}$ belong to $\Delta$ as well. A columnconvex diagram is defined analogously. A diagram is convex if it is both row-convex and column-convex.

A convex diagram is said to be bottom-justified if its bottom row intersects each of its columns, and it is said to be right-justified if its rightmost column intersects each of its rows. 
For our purposes, we need to deal with two types of diagrams, known as Ferrers diagrams and stack diagrams. A Ferrers diagram (also known as Ferrers shape) is a convex diagram that is bottom-justified and right-justified. A stack diagram is a convex bottom-justified diagram.

Our convention of drawing Ferrers diagrams as right-justified rather than left-justified shapes is different from standard practice; however, our definition will be more intuitive in the context of our applications.

Clearly, every Ferrers diagram is also a stack diagram. On the other hand, a stack diagram can be regarded as a union of a Ferrers diagram and a vertically reflected copy of another Ferrers diagram.

A filling of a diagram $\Delta$ is an assignment that inserts into each cell of the diagram a value 0 or 1 . In such filling, a 0 -cell is a cell that is filled with value 0 , and a 1-cell is filled with value 1. A filling is a transversal if each of its columns and each of its rows contains exactly one 1-cell. A filling is sparse if every column and every row has at most one 1-cell. A column of a filling is a zero column if it contains no 1-cell. A zero row is defined analogously.

A matrix with entries equal to 0 or 1 will be considered as a special case of a filling, whose underlying diagram is a rectangle. We will now introduce a correspondence between sequences of integers and matrices. This correspondence will provide a link between pattern avoidance in matchings and pattern avoidance in fillings.

Let $s=s_{1} s_{2} \cdots s_{n}$ be a sequence of positive integers, none of them greater than $k$. We let $M(s, k)$ denote the $0-1$ matrix with $k$ rows and $n$ columns with the property that the column $i$ has a unique 1-cell, and this 1-cell appears in row $s_{i}$.

In the special case when $s$ is a permutation of order $n$, then $M(s, n)$ is known as the permutation matrix of $s$.

Let us stress that in the definition of $M(s, k)$, we do not assume that $s$ contains all the integers $1, \ldots, k$. Thus, the matrix $M(s, k)$ might have zero rows.

Among several possibilities to define pattern avoidance in fillings, the following approach seems to be the most useful and most common.

Definition 4.1. Let $M=\left(m_{i j} ; i \in[r], j \in[c]\right)$ be a matrix with $r$ rows and $c$ columns with all entries equal to 0 or 1 , and let $F$ be a filling of a diagram $\Delta$. We say that $F$ contains $M$ if $F$ contains $r$ distinct rows $i_{1}<\cdots<i_{r}$ and $c$ distinct columns $j_{1}<\cdots<j_{c}$ with the following two properties.

- Each of the rows $i_{1}, \ldots, i_{r}$ intersects all columns $j_{1}, \ldots, j_{c}$ in a cell of $\Delta$.

- If $m_{k \ell}=1$ for some $k$ and $\ell$, then the cell in row $i_{k}$ and column $j_{\ell}$ of $F$ is a 1-cell.

If $F$ does not contain $M$, we say that $F$ avoids $M$. We will say that two 01-matrices $M$ and $M^{\prime}$ are shape-Wilf equivalent (denoted by $M \stackrel{\mathrm{sW}}{\sim} M^{\prime}$ ) if for every Ferrers diagram $\Delta$, there is a bijection $\phi$ between $M$-avoiding and $M^{\prime}$-avoiding sparse fillings of $\Delta$, with the property that an $M$-avoiding filling $F$ has the same zero rows and zero columns as its image $\phi(F)$. 
If $p$ and $q$ are permutations of the same order $n$, we say that $p$ and $q$ are shape-Wilf equivalent if their corresponding permutation matrices $M(p, n)$ and $M(q, n)$ are shapeWilf equivalent.

It is not hard to see that if $M$ and $M^{\prime}$ have no zero rows and zero columns, then $M$ and $M^{\prime}$ are shape-Wilf equivalent if and only if for each Ferrers diagram $\Delta$ there is a bijection between $M$-avoiding and $M^{\prime}$-avoiding transversals of $\Delta$.

The concept of shape-Wilf equivalence (restricted to permutations) has been applied in the study of pattern avoidance in permutations $[1,13]$.

\subsection{Partial matchings and fillings of Ferrers diagrams}

The idea that a filling of a Ferrers diagram could be used to represent a matching is not new. It has been shown by A. de Mier [6] that matchings are in bijection with transversals of Ferrers shapes, and Krattenthaler [10] has used fillings of Ferrers diagram as a tool to construct a bijection between $k$-nonnesting and $k$-noncrossing matchings. We push the idea further, and show how to apply the concept of shape-Wilf equivalence to obtain bijections between more general pattern avoiding classes.

Lemma 4.2. Let $k>0$ be an integer. Let $\rho$ be a (possibly empty) canonical sequence representing a partial matching. Let $s$ and $s^{\prime}$ be two sequences over the alphabet $[k]$, such that each symbol from $[k]$ appears at most once in $s$ and at most once in $s^{\prime}$. If the matrices $M(s, k)$ and $M\left(s^{\prime}, k\right)$ are shape-Wilf equivalent, then the two partial matchings $\pi=12 \cdots k(\rho+k) s$ and $\pi^{\prime}=12 \cdots k(\rho+k) s^{\prime}$ are equivalent.

The main argument used in the proof of Lemma 4.2 is inspired by an idea that has been previously used by the authors in the study of pattern avoiding set partitions [7]. However, the previous arguments required stronger assumptions about $s$ and $s^{\prime}$ than just shape-Wilf equivalence. In fact, some partial patterns whose equivalence follows from Lemma 4.2 (e.g., 1234213 and 1234132) are not partition-equivalent.

Proof of Lemma 4.2. Let $\ell$ be the largest element of $\rho$. Define the matrix $R=M(\rho, \ell)$.

Let $\mu=\mu_{1} \mu_{2} \cdots \mu_{2 n}$ be a matching with $n$ edges. Consider the matrix $M=M(\mu, n)$. We will distinguish two types of cells of $M$, which we will call the red cells and the green cells. A cell in row $i$ and column $j$ is green, if it satisfies the following two conditions:

1. The submatrix of $M$ induced by the rows $i+1, i+2, \ldots, n$ and the columns $1, \ldots, j-1$ contains $R$.

2. At least one 1-cell in row $i$ appears strictly to the left of column $j$.

A cell is red if it is not green. Notice that if $\rho$ is a nonempty sequence (and hence $R$ is a nonempty matrix), then the first of the two conditions above actually implies the second one, because of the properties of canonical sequences. On the other hand, if $\rho$ is empty, then the first condition holds trivially for any cell, and the second condition guarantees that the green cells of a given row $i$ are exactly the cells that are to the right 
of the leftmost 1-cell in row $i$. In both cases, the leftmost 1-cell in a given row is never green, so each row has at most one green 1-cell.

Observe that the green cells of $M$ form a sparse filling of a (possibly empty) Ferrers diagram. Let $G(M)$ denote this 'green' filling. Let $s$ and $s^{\prime}$ be sequences satisfying the assumptions of the lemma. It can be routinely checked that the filling $G(M)$ avoids the matrix $M(s, k)$ if and only if $\mu$ avoids the pattern $\pi=12 \cdots k(\rho+k) s$.

Let us now describe the bijection between $\pi$-avoiding and $\pi^{\prime}$-avoiding matchings. Suppose that the matrices $S=M(s, k)$ and $S^{\prime}=M\left(s^{\prime}, k\right)$ are shape-Wilf equivalent via a bijection $\phi$ that transforms $S$-avoiding sparse fillings to $S^{\prime}$-avoiding sparse fillings of the same diagram, while preserving the zero rows and zero columns.

Consider a $\pi$-avoiding matching $\mu \in \mathcal{M}_{n}(\pi)$. Define the matrix $M$ and the filling $G=G(M)$ as above. We know that $G$ avoids $S$. Consider now the $S^{\prime}$-avoiding filling $G^{\prime}=\phi(G)$. Create a matrix $M^{\prime}$ from $M$ by inserting the values of $G^{\prime}$ into the green cells of $M$. By construction, each row of $M^{\prime}$ has two 1-cells, and each column has one 1-cell. Moreover, the leftmost 1-cell in each row of $M$ is a red cell and hence its value is not modified by the transform, which means that it is also the leftmost 1-cell of the corresponding row of $M^{\prime}$. Consequently, there is a matching $\mu^{\prime} \in \mathcal{M}_{n}$ such that $M^{\prime}=$ $M\left(\mu^{\prime}, n\right)$.

Assume that the cells of $M^{\prime}$ are colored red and green by the two rules introduced before. We claim that a cell is green in $M^{\prime}$ if and only if it is green in $M$. Let $M(\geqslant i,<j)$ denote the submatrix of $M$ induced by rows $i, i+1, \ldots, n$ and columns $1,2 \ldots, j$. Note that the color of the cell $(i, j)$ in the matrix $M$ only depends on the values in $M(\geqslant i,<j)$. If the cell $(i, j)$ is red in $M$, then all the cells of $M(\geqslant i,<j)$ are also red in $M$. Since each red cell of $M$ has the same value in $M$ as in $M^{\prime}$, it follows that $M(\geqslant i,<j)=M^{\prime}(\geqslant i,<j)$, and hence $(i, j)$ is also red in $M^{\prime}$. In other words, each red cell of $M$ remains red in $M^{\prime}$.

Suppose now that $(i, j)$ is the leftmost green cell of $M$ in row $i$. This implies that $M(\geqslant i,<j)$ only contains red cells of $M$, and thus $M(\geqslant i,<j)=M^{\prime}(\geqslant i,<j)$, which implies that $(i, j)$ must also be green in $M^{\prime}$. Every cell on row $i$ to the right of $(i, j)$ must then also be green in $M^{\prime}$, which shows that all the green cells of $M$ are also green in $M^{\prime}$, as claimed.

Now that we have seen that the mapping $M \mapsto M^{\prime}$ preserves the color of the cells, we know that $G^{\prime}$ corresponds exactly to the green cells of $M^{\prime}$, i.e., $G^{\prime}=G\left(M^{\prime}\right)$. Since $G^{\prime}$ avoids $S^{\prime}$, we know that $\mu^{\prime}$ avoids $\pi^{\prime}$. Obviously, the transform from $\pi$ to $\pi^{\prime}$ can be inverted, and gives a bijection between $\mathcal{M}_{n}(\pi)$ and $\mathcal{M}_{n}\left(\pi^{\prime}\right)$.

We now plug previous results on shape-Wilf equivalence into Lemma 4.2, to obtain the following corollary.

Corollary 4.3. For any partial matching $\rho$, the two patterns

$$
123(\rho+3) 213 \text { and } 123(\rho+3) 132
$$

are equivalent.

Proof. As shown by Stankova and West [13], the two permutation matrices $M(213,3)$ and $M(132,3)$ are shape-Wilf equivalent. The corollary then follows from Lemma 4.2. 
In fact, there are more results on shape-Wilf equivalence in the literature than the above-mentioned result of Stankova and West. For instance, Backelin et al. [1] have shown that for any $k \geqslant \ell \geqslant 1$ the permutation $k(k-1) \cdots(\ell+1) 12 \cdots \ell$ is shape-Wilf equivalent to the identity permutation $12 \cdots k$. Apart from that, they have observed that if $p$ and $q$ are shape-Wilf equivalent permutations of order $n$ and $r$ is an arbitrary permutation, then the two permutations $(r+n) p$ and $(r+n) q$ are shape-Wilf equivalent as well.

However, any equivalences of partial matchings that we may deduce from these results using Lemma 4.2 already follow from Fact 2.1 .

\subsection{Partial matchings and fillings of stack diagrams}

We now describe a more technical argument, which allows to translates certain bijections between fillings of stack diagrams into bijections between pattern avoiding matchings.

Let $\Pi$ be a stack diagram. The content of $\Pi$ is the sequence of the column heights of $\Pi$, listed in weakly decreasing order.

Let $\Pi$ be a stack diagram with $r$ rows. For $i \in[r]$, let $L_{i}(\Pi)$ and $R_{i}(\Pi)$ be the column indices of the leftmost and rightmost cells of the $i$-th row of $\Pi$. Note that $L_{1}(\Pi) \leqslant$ $L_{2}(\Pi) \leqslant \cdots \leqslant L_{r}(\Pi)$, and symmetrically $R_{1}(\Pi) \geqslant R_{2}(\Pi) \geqslant \cdots \geqslant R_{r}(\Pi)$. Let $i>1$ be a row of $\Pi$ such that $R_{i}(\Pi)<R_{i-1}(\Pi)$. The right shift of $\Pi$ at height $i$ is the stack diagram $\Pi^{\prime}$ satisfying

- $L_{j}\left(\Pi^{\prime}\right)=L_{j}(\Pi)$ and $R_{j}\left(\Pi^{\prime}\right)=R_{j}(\Pi)$ for every $j<i$, and

- $L_{j}\left(\Pi^{\prime}\right)=L_{j}(\Pi)+1$ and $R_{j}\left(\Pi^{\prime}\right)=R_{j}(\Pi)+1$ for every $j \geqslant i$.

Intuitively, $\Pi^{\prime}$ is obtained from $\Pi$ by shifting each cell in row $i$ and above by one column to the right. Note that $\Pi^{\prime}$ and $\Pi$ have the same content.

Let $\Pi$ be again a stack diagram with $r$ rows and let $Z \subseteq[r]$ be a set of row-indices of $\Pi$. Let $P$ be a sparse matrix. Let $\mathcal{F}(P, Z, \Pi)$ denote the set of all the fillings $F$ of $\Pi$ that have the following properties.

- $F$ avoids $P$.

- For each $i \in Z$, the $i$-th row of $F$ is a zero row.

- For each $i \in[r] \backslash Z$, the $i$-th row of $F$ has exactly one 1-cell.

- Each column of $F$ has exactly one 1-cell.

Let $P$ be a sparse matrix. Let $\Pi$ be a stack diagram with $r$ rows, and let $i>1$ be a row-index such that $R_{i}(\Pi)<R_{i-1}(\Pi)$. Let $\Pi^{\prime}$ be the right shift of $\Pi$ at height $i$. We say that $P$ is shift-proof for $\Pi$ at height $i$ if for every set $Z \subseteq[r]$ that contains $i$, there is a bijection $\phi$ between $\mathcal{F}(P, Z, \Pi)$ and $\mathcal{F}\left(P, Z, \Pi^{\prime}\right)$. Notice that in this definition, we make no assumption about fillings that contain 1-cells in row $i$. 
We say that a sparse matrix $P$ is shift-proof if for every stack diagram $\Pi$ and every row-index $i>0$ such that $R_{i}(\Pi)<R_{i-1}(\Pi), P$ is shift-proof for $\Pi$ at height $i$. The concept of shift-proofness is motivated by the following theorem.

Theorem 4.4. Let $s=s_{1} \cdots s_{\ell}$ be a sequence over the alphabet $[k]$ that contains each number at most once. If the matrix $M(s, k)$ is shift-proof, then the patterns $\sigma=12 \cdots k(k+1) s$ and $\rho=12 \cdots k s(k+1)$ are partial-matching equivalent.

Fix an $n \geqslant 1$ and a sequence of block sizes $a=\left(a_{1}, \ldots, a_{n}\right)$, with $a_{i} \in\{1,2\}$. Let $P(a)$ be the set of partial matchings whose $i$-th block has size $a_{i}$, or equivalently, the set of canonical sequences with $a_{i}$ occurrences of the symbol $i$. Let $N$ denote the sum $\sum_{i} a_{i}$. Recall that $P(\sigma ; a)$ is the sets of $\sigma$-avoiding elements of $P(a)$, and $p(\sigma ; a)$ is the cardinality of $P(\sigma ; a)$.

Fix a sequence $s$ satisfying the assumptions of Theorem 4.4, and let $\sigma$ and $\rho$ be the two patterns from the theorem's statement. We will prove Theorem 4.4 by constructing a bijection between $P(\sigma ; a)$ and $P(\rho ; a)$. Before we state the proof, we need several auxiliary statements describing the structure of the partial matchings in the two pattern-avoidance classes.

We first focus on the class $P(\sigma ; \tau)$. Let $\mu=\mu_{1} \cdots \mu_{N}$ be a partial matching from $P(a)$. We say that an element $\mu_{i}$ is left-dominating if $\mu_{i} \geqslant \mu_{j}$ for each $j<i$.

Assume now that $\mu_{j}$ is an element of $\mu$ that is not left-dominating. We shall say that such an element is left-dominated. For left-dominated element $\mu_{j}$, let $i$ be the largest index such that $i<j$ and $\mu_{i}$ is left-dominating. We then say that $\mu_{i}$ left-dominates $\mu_{j}$. It is easy to see that such an index $i$ always exists, and that $\mu_{i}>\mu_{j}$.

The left shadow of $\mu$ is the sequence $\widehat{\mu}$ obtained by replacing each left-dominated element of $\mu$ by the symbol ' $*$ '. We will say that a non-star symbol $j$ left-dominates an occurrence of a star, if $j$ is the rightmost non-star to the left of the star.

For example, if $\mu=12321434$, the left shadow of $\mu$ is the sequence $\widehat{\mu}=123 * * 4 * 4$. In $\widehat{\mu}$, the symbol ' 3 ' left-dominates two stars, and the first occurrence of ' 4 ' left-dominates one star. No other symbol dominates any star.

Left-shadow sequences of partial matchings from the set $P(a)$ are characterized by the next observation, whose proof we omit.

Observation 4.5. Let $\widehat{\mu}$ be a sequence of length $N$ over the alphabet $\{1,2, \ldots, n, *\}$. For $k \in[n]$ let $m_{k}$ be the number of occurrences of $k$ in $\widehat{\mu}$, and let $d_{k}$ be the total number of stars dominated by the occurrences of $k$ in $\widehat{\mu}$. Then $\widehat{\mu}$ is the left shadow of a partial matching from $P(a)$ if and only if it satisfies the following conditions.

1. The non-star symbols of $\widehat{\mu}$ form a weakly increasing subsequence.

2. For every $k \in[n], 1 \leqslant m_{k} \leqslant a_{k}$.

3. Each star is dominated by a non-star (i.e., the leftmost symbol of $\widehat{\mu}$ is not a star).

4. For every $k \in[n]$,

$$
\sum_{i<k} m_{i}+\sum_{j \leqslant k} d_{j} \leqslant \sum_{i<k} a_{i}
$$


We say that a sequence $\widehat{\mu}$ is a left-shadow sequence, if it satisfies the conditions of Observation 4.7 .

Definition 4.6. Let $\mu \in P(a)$ be a partial matching. Let $F=F(\mu)$ be the sparse filling of a Ferrers diagram defined by the following conditions.

1. The columns of $F$ correspond to the left-dominated elements of $\mu$. The $i$-th column of $F$ has height $j$ if the $i$-th left-dominated element of $\mu$ is dominated by an occurrence of $j+1$.

2. The $i$-th column of $F$ has a 1-cell in row $r$ if the $i$-th left-dominated element of $\mu$ is equal to $r$.

Note that for $\mu \in P(a)$, the filling $F(\mu)$ is in fact a subdiagram of the matrix $M(\mu, n)$. As an example, consider the matching $\mu=123442516563$, with its left shadow $\widehat{\mu}=$ $12344 * 5 * 6 * 6 *$. The filling $F(\mu)$ is depicted on Figure 2 .
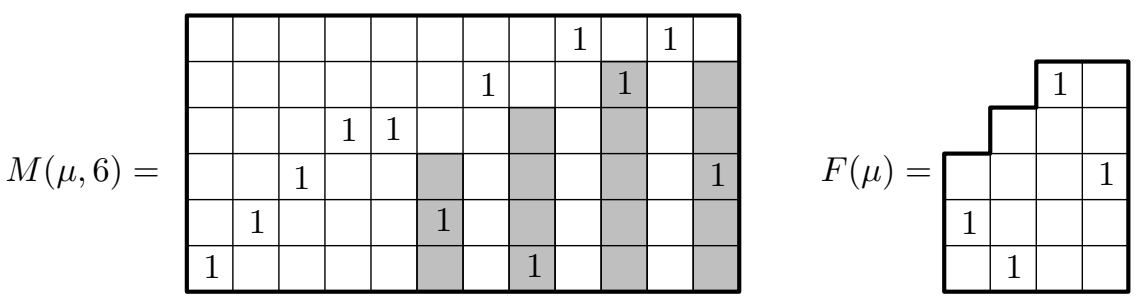

Figure 2: An example of the matrix $M(\mu, n)$ and the filling $F(\mu)$, for $\mu=123442516563$, with left shadow $\widehat{\mu}=12344 * 5 * 6 * 6 *$. The 0 -cells are represented by empty boxes. The shaded cells of the matrix correspond to the cells of $F(\mu)$.

Let $\Delta(\mu)$ be the underlying Ferrers diagram of the filling $F(\mu)$. Note that $\Delta(\mu)$ is in fact uniquely determined by the left shadow $\widehat{\mu}$ of $\mu$. More precisely, the number of columns of height $h$ in $\Delta(\mu)$ is equal to the number of stars in the left shadow $\widehat{\mu}$ that are dominated by an occurrence of $h+1$. We may thus write $\Delta(\widehat{\mu})$ for the Ferrers diagram determined by $\widehat{\mu}$.

By definition, every column of $F(\mu)$ has exactly one 1-cell. Note that the $i$-th row of the filling $F(\mu)$ is a zero row if and only if the left shadow $\widehat{\mu}$ has $a_{i}$ occurrences of the symbol $i$. This means that the zero rows of the $F(\mu)$ are uniquely determined by $\widehat{\mu}$. Let $Z(\widehat{\mu})$ be the set of zero rows of $F$.

It is clear that the left shadow $\widehat{\mu}$ and the filling $F(\mu)$ together uniquely determine $\mu$. In fact, for every sparse filling $F^{\prime}$ of $\Delta(\widehat{\mu})$ with the set of zero rows $Z(\widehat{\mu})$ there is a unique $\mu^{\prime} \in P(a)$ with left shadow $\widehat{\mu}$ satisfying $F\left(\mu^{\prime}\right)=F^{\prime}$. (Note that any sparse filling of $\Delta(\widehat{\mu})$ with the set of zero rows $Z(\widehat{\mu})$ must have a 1-cell in each column.) Thus, for any left-shadow sequence $\widehat{\mu}$, there is a one-to-one correspondence between partial matchings from $P(a)$ with left shadow $\widehat{\mu}$ and sparse fillings of $\Delta(\widehat{\mu})$ with zero rows $Z(\widehat{\mu})$.

The following observation is a straightforward application of the terminology introduced above. We omit its proof. 
Observation 4.7. Assume that $s=s_{1} \cdots s_{\ell}$ is a sequence of distinct numbers from the set $[k]$. A partial matching $\mu \in P(a)$ avoids the pattern $12 \cdots k(k+1) s$ if and only if the filling $F(\mu)$ avoids the matrix $M(s, k)$.

We now focus on partial matchings that avoid the pattern $\rho=12 \cdots k s(k+1)$, where $s$ is again a sequence of distinct integers from $[k]$.

Let $\nu \in P(a)$ be a partial matching. We say that an element $\nu_{i}$ of $\nu$ is right-dominating if either $\nu_{i}>\nu_{j}$ for each $j>i$, or $\nu_{i}>\nu_{j}$ for each $j<i$. If $\nu_{i}$ is not right-dominating, we say that it is right-dominated. We say that $\nu_{i}$ right-dominates $\nu_{j}$ if $\nu_{i}$ is the leftmost right-dominating element appearing to the right of $\nu_{j}$, and $\nu_{j}$ itself is not right-dominating.

The right shadow $\widetilde{\nu}$ of a $\nu$ is obtained by replacing each right-dominated element of $\nu$ by a star.

For example, the right shadow of $\nu=1213454523$ is the sequence $12 * 345 * 5 * 3$. In this sequence, each of the two occurrences of the symbol ' 3 ' right-dominates one star, and the second occurrence of ' 5 ' also right-dominates one star. Right shadows are characterized by the next observation.

Observation 4.8. Let $\widetilde{\nu}$ be a sequence of length $N$ over the alphabet $\{1,2, \ldots, n, *\}$. For $k \in[n]$, let $m_{k}$ be the number of occurrences of $k$ in $\widetilde{\nu}$, and let $d_{k}$ be the total number of stars dominated by the occurrences of $k$ in $\widetilde{\nu}$. Then $\widetilde{\nu}$ is the right shadow of a partial matching from $P(a)$ if and only if it satisfies the following conditions.

1. The non-star symbols of $\widetilde{\nu}$ form a sequence of the form $12 \cdots n x_{1} x_{2} \cdots x_{m}$, where $x_{1} x_{2} \cdots x_{m}$ is a strictly decreasing sequence of numbers from $[n]$.

2. For every $k \in[n], 1 \leqslant m_{k} \leqslant a_{k}$.

3. Each star is dominated by a non-star (i.e., the rightmost symbol of $\widetilde{\nu}$ is not a star).

4. For every $k \leqslant n$,

$$
\sum_{i<k} m_{i}+\sum_{j \leqslant k} d_{j} \leqslant \sum_{i<k} a_{i}
$$

A sequence $\widetilde{\nu}$ satisfying the conditions of Observation 4.8 is a right-shadow sequence.

Suppose that $\widetilde{\nu}$ is a right-shadow sequence and $\widehat{\mu}$ is a left-shadow sequence. We say that $\widehat{\mu}$ and $\widetilde{\nu}$ are twins if for every $i \in[n]$, the next two conditions are satisfied.

- The symbol $i$ has the same number of occurrences in $\widehat{\mu}$ as in $\widetilde{\nu}$.

- The number of stars left-dominated by each occurrence of $i$ in $\widehat{\mu}$ is equal to the number of stars right-dominated by the corresponding occurrence of $i$ in $\widetilde{\nu}$.

From Observations 4.5 and 4.8, we deduce that every left-shadow sequence has a unique right-shadow twin and vice versa. For instance, consider the left-shadow sequence $\widehat{\mu}=$ $1233 * 45 * * 5$. The second occurrence of 3 in $\widehat{\mu}$ left-dominates a single star, and the first occurrence of 5 left-dominates two stars. Thus, the twin of $\widehat{\mu}$ is the right-shadow sequence $\widetilde{\nu}=1234 * * 55 * 3$. 
Let $\nu \in P(a)$ be a partial matching. Let $S=S(\nu)$ be the sparse filling of a stack diagram defined by the following conditions.

1. The columns of $S$ correspond to the right-dominated elements of $\nu$. The $i$-th column of $S$ has height $j$ if the $i$-th right-dominated element of $\nu$ is dominated by an occurrence of $j+1$.

2. The $i$-th column of $S$ has a 1-cell in row $j$ if the $i$-th right-dominated element of $\nu$ is equal to $j$.

For example, $\nu=123415563624$ has the right shadow $\widetilde{\nu}=1234 * 5 * 6 * 6 * 4$. Figure 3 shows the filling $S(\nu)$, which is a subfilling of the matrix $M(\nu, 6)$. Notice that $\widetilde{\nu}$ is the twin of the left shadow $\widehat{\mu}$ from Figure 2 .
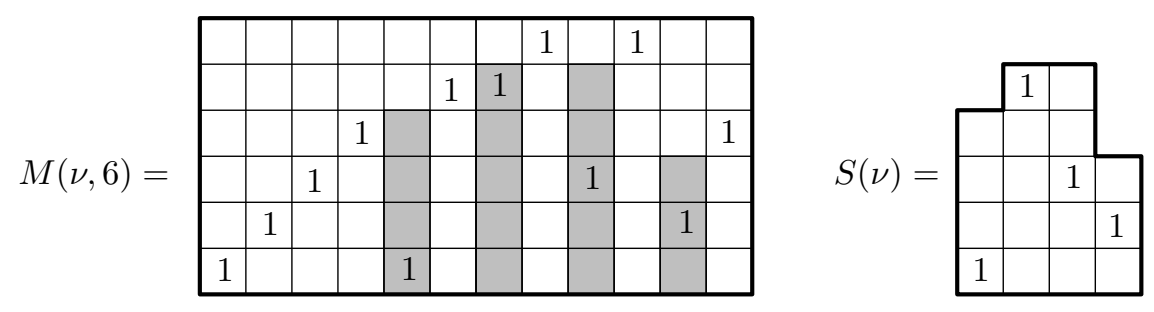

Figure 3: The filling $S(\nu)$ associated with $\nu=123415563624$. The right shadow of $\nu$ is $\widetilde{\nu}=1234 * 5 * 6 * 6 * 4$.

Let $\Sigma(\nu)$ be the underlying diagram of $S(\nu)$. Notice that $\Sigma(\nu)$ is uniquely determined by the right shadow $\widetilde{\nu}$ of the matching $\nu$, so we may write $\Sigma(\widetilde{\nu})$ for the diagram determined by $\widetilde{\nu}$. The $i$-th row of $S(\nu)$ is a zero row if and only if $\widetilde{\nu}$ has $a_{i}$ occurrences of the symbol $i$, which shows that the set of zero rows of $S(\nu)$ is determined by $\widetilde{\nu}$. Let $Z(\widetilde{\nu})$ be the set of zero rows of $S(\nu)$.

The sequence $\widetilde{\nu}$ and the filling $S(\nu)$ together determine $\nu$. This establishes, for any right-shadow sequence $\widetilde{\nu}$, a bijection between the partial matchings from $P(a)$ with right shadow $\widetilde{\nu}$ and the sparse fillings of $\Sigma(\widetilde{\nu})$ with the set of zero rows $Z(\widetilde{\nu})$.

The following observation follows directly from our definitions, and we again omit its proof.

Observation 4.9. Assume that $s=s_{1} \cdots s_{\ell}$ is a sequence of distinct numbers from the set $[k]$. A matching $\nu$ avoids the pattern $\rho=12 \cdots k s(k+1)$ if and only if the filling $S(\nu)$ avoids $M(s, k)$.

Lemma 4.10. Let $\widetilde{\nu}$ be the right shadow of a sequence $\nu \in P(a)$, and let $\Sigma=\Sigma(\widetilde{\nu})$ and $S=S(\nu)$ be as above. If, for some row index $i>1$ of $\Sigma$ we have $R_{i}(\Sigma)<R_{i-1}(\Sigma)$, then $\widetilde{\nu}$ has two occurrences of the symbol $i$, and hence $i$ is a zero row of $S$.

Proof. Let $h$ be the height of the column $R_{i}(\Sigma)$ of $\Sigma$. Clearly $h \geqslant i$. Suppose that $R_{i}(\Sigma)<R_{i-1}(\Sigma)$. This means that the column $R_{i}(\Sigma)+1$ of $\Sigma$ has height $i-1$. This 
in turn means that the right shadow $\widetilde{\nu}$ has an occurrence of the symbol $i$ that follows an occurrence of the symbol $h+1>i$. Apart from that, $\widetilde{\nu}$ must also contain another occurrence of $i$, which is to the left of any occurrence of $h+1$, because the first $n$ non-star symbols of $\widetilde{\nu}$ form the sequence $12 \cdots n$. Thus, $\widetilde{\nu}$ has two occurrences of $i$, and hence the $i$-th row of $S$ is a zero row.

We are now ready to prove Theorem 4.4. Let $s=s_{1} s_{2} \cdots s_{\ell}$ be a sequence of distinct integers from the set $[k]$, and assume that the matrix $P=M(s, k)$ is shift-proof. Recall that $\sigma$ is the pattern $12 \cdots k(k+1) s$ and $\rho$ is the pattern $12 \cdots k s(k+1)$. Our goal is to construct a bijection between $P(\sigma ; a)$ and $P(\rho ; a)$.

It suffices to show that for each pair of twins $\widehat{\mu}$ and $\widetilde{\nu}$, where $\widehat{\mu}$ is a left shadow and $\widetilde{\nu}$ is a right shadow, the number of partial matchings in $P(\sigma ; a)$ with left shadow $\widehat{\mu}$ is equal to the number of partial matchings in $P(\rho ; a)$ with right shadow $\widetilde{\nu}$.

Let $\widehat{\mathcal{M}}$ and $\widehat{\mathcal{M}}$ denote, respectively, the set of elements in $P(\sigma ; a)$ with left shadow $\widehat{\mu}$, and the set of elements of $P(\rho ; a)$ with right shadow $\widetilde{\nu}$. Let $\Delta=\Delta(\widehat{\mu})$ and $\Sigma=\Sigma(\widetilde{\nu})$. Since $\widehat{\mu}$ and $\widetilde{\nu}$ are twins, the diagrams $\Delta$ and $\Sigma$ have the same content. Let $r$ be the number of rows of $\Delta$ (and of $\Sigma$ ).

Define the set $Z=\left\{i \leqslant r: \widehat{\mu}\right.$ has $a_{i}$ occurrences of $\left.i\right\}$. Note that $Z$ is also equal to the set $\left\{i \leqslant r: \widetilde{\nu}\right.$ has $a_{i}$ occurrences of $\left.i\right\}$. From Observations 4.7 and 4.9, we deduce that the elements of $\widehat{\mathcal{M}}$ correspond bijectively to fillings from the set $\mathcal{F}(P, Z, \Delta)$ and the elements of $\widetilde{\mathcal{M}}$ correspond bijectively to fillings from $\mathcal{F}(P, Z, \Sigma)$.

We now show that there is a bijection between $\mathcal{F}(P, Z, \Delta)$ and $\mathcal{F}(P, Z, \Sigma)$. Since $\Sigma$ and $\Delta$ have the same content, and since $\Delta$ is a right-justified diagram, we can find a sequence of stack diagrams $\Pi_{0}, \Pi_{1}, \ldots, \Pi_{k}$ such that $\Sigma=\Pi_{0}, \Delta=\Pi_{k}$, and for any $j \geqslant 1$, $\Pi_{j}$ is a right shift of $\Pi_{j-1}$ at a height $h_{j}$. Moreover, by Lemma 4.10, each $h_{j}$ belongs to $Z$. Since $P$ is assumed to be shift-proof, we know that $\left|\mathcal{F}\left(P, Z, \Pi_{j-1}\right)\right|=\left|\mathcal{F}\left(P, Z, \Pi_{j}\right)\right|$ for each $j \in[k]$, and hence $|\mathcal{F}(P, Z, \Sigma)|=|\mathcal{F}(P, Z, \Delta)|$. This proves Theorem 4.4.

To apply Theorem 4.4, we need examples of shift-proof patterns. The only permutation matrices known to be shift-proof are the identity matrices $M(12 \cdots \ell, \ell)$ and the antidiagonal matrices $M(\ell(\ell-1) \cdots 1, \ell)$. More generally, for any $k \geqslant \ell$, both $M(12 \cdots \ell, k)$ and $M(\ell(\ell-1) \cdots 1, k)$ are shift-proof. This follows directly from more general results of Rubey related to fillings of moon diagrams [11, Theorem 5.3]. We state this as a fact.

Fact 4.11. For any $k \geqslant \ell \geqslant 1$, the matrices $M(12 \cdots \ell, k)$ and $M(\ell(\ell-1) \cdots 1, k)$ are shift-proof.

Combining Fact 2.1, Lemma 3.4, Theorem 4.4 and Fact 4.11, we get the following result.

Corollary 4.12. Let $k, \ell$, and $m$ be integers, with $k \geqslant \ell \geqslant m$. The following four partial patterns are partial-matching equivalent:

- $12 \cdots k m(m+1) \cdots \ell(k+1)$,

- $12 \cdots k(k+1) m(m+1) \cdots \ell$, 
- $12 \cdots k \ell(\ell-1) \cdots m(k+1)$, and

- $12 \cdots k(k+1) \ell(\ell-1) \cdots m$.

We found two more examples of shift-proof matrices, presented in the next lemma.

Lemma 4.13. The matrix $P=M(13,3)$ and the matrix $P^{\prime}=M(31,3)$ are both shiftproof.

Proof. It is enough to prove the lemma for $P$, the argument for $P^{\prime}$ is symmetric.

Let $\Pi$ be a stack diagram with $r$ rows and $c$ columns. Let $i>1$ be a row index such that $R_{i}(\Pi)<R_{i-1}(\Pi)$. Let $\Pi^{\prime}$ be the right shift of $\Pi$ at height $i$. Let $Z$ be a set of row-indices containing $i$.

Fix a filling $F \in \mathcal{F}(P, Z, \Pi)$. We will say that a cell of $F$ is high if it belongs to a row $i^{\prime}>i$, and a cell is low if it belongs to a row $i^{\prime}<i$. Note that each column of $F$ has exactly one 1-cell, and each 1-cell is either high or low, because row $i$ is a zero row by assumption. We may thus partition the set $[c]$ of column indices into two sets $\operatorname{High}(F)$ and $\operatorname{Low}(F)$, defined as

$$
\begin{aligned}
\operatorname{High}(F) & =\{j: \text { column } j \text { contains a high 1-cell }\}, \text { and } \\
\operatorname{Low}(F) & =\{j: \text { column } j \text { contains a low 1-cell }\} .
\end{aligned}
$$

Let $\ell$ be the cardinality of $\operatorname{Low}(F)$, and let $j_{1}<j_{2}<\cdots<j_{\ell}$ be the elements of Low $(F)$.

We now describe how to transform the filling $F$ into a filling $F^{\prime}$ from $\mathcal{F}\left(P, Z, \Pi^{\prime}\right)$. For any $i^{\prime} \geqslant i$, the row $i^{\prime}$ of $F^{\prime}$ contains the same values as the row $i^{\prime}$ of $F$, only shifted to the right by one cell. This implies that $j \in \operatorname{High}\left(F^{\prime}\right)$ if and only if $j-1 \in \operatorname{High}(F)$, and consequently $j \in \operatorname{Low}\left(F^{\prime}\right)$ if and only if $j=1$ or $j-1 \in \operatorname{Low}(F)$. Let $j_{1}^{\prime}<j_{2}^{\prime}<\cdots<j_{\ell}^{\prime}$ be the elements of Low $\left(F^{\prime}\right)$. It remains to specify the values of $F^{\prime}$ in the rows below row $i$. Necessarily, all the 1-cells in these rows can only appear in the columns from $\operatorname{Low}\left(F^{\prime}\right)$. For any $i^{\prime}<i$, the row $i^{\prime}$ of $F^{\prime}$ has a 1-cell in a column $j_{m}^{\prime} \in \operatorname{Low}\left(F^{\prime}\right)$ if and only if the row $i^{\prime}$ of $F$ has a 1-cell in column $j_{m}$. This determines $F^{\prime}$ uniquely.

Notice that if $j$ is a column of $F$ with fewer than $i-1$ cells, then $j$ has the same height and the same values in $F$ as in $F^{\prime}$.

Let us show that $F^{\prime}$ belongs to $\mathcal{F}\left(P, Z, \Pi^{\prime}\right)$. By construction, $F^{\prime}$ has exactly one 1 -cell in each column, and at most one 1-cell in each row. Also, $\Pi^{\prime}$ has the same zero rows as $\Pi$.

It remains to show that $F^{\prime}$ avoids $P$. Assume for contradiction that $F^{\prime}$ contains a copy of $P$ induced by columns $c_{1}<c_{2}$ and rows $r_{1}<r_{2}<r_{3}$. If both 1-cells in this copy of $P$ are high, then $F$ contains a copy of $P$ in columns $c_{1}-1$ and $c_{2}-1$. If, on the other hand, both of the 1-cells are low, then $c_{1}=j_{a}^{\prime}$ and $c_{2}=j_{b}^{\prime}$ for some $a<b$. In such case, $F$ contains a copy of $P$ in columns $j_{a}$ and $j_{b}$. Finally, assume that $c_{1} \in \operatorname{Low}\left(F^{\prime}\right)$ and $c_{2} \in \operatorname{High}\left(F^{\prime}\right)$. Then row $r_{3}$ is above row $i$. Since the column $c_{1}$ intersects row $r_{3}$, we see that $c_{1}>1$. Consequently, $c_{1}-1$ intersects row $r_{3}$ in $F$, and $c_{1}-1$ belongs to Low $(F)$. We conclude that $F$ has a 1-cell in row $r_{3}>i$ and column $c_{2}-1$, and another 1-cell in column $c_{1}-1$ and a row smaller than $i$. This shows that the two columns $c_{1}-1$ and 
$c_{2}-1$ of $F$ contain a copy of $P$. In all cases we have a contradiction, showing that $F^{\prime}$ belongs to $\mathcal{F}\left(P, Z, \Pi^{\prime}\right)$.

By analogous arguments, we can show that every filling $F^{\prime} \in \mathcal{F}\left(P, Z, \Pi^{\prime}\right)$ is obtained from a unique filling $F \in \mathcal{F}(P, Z, \Pi)$. Thus, the mapping $F \mapsto F^{\prime}$ is a bijection between $\mathcal{F}(P, Z, \Pi)$ and $\mathcal{F}\left(P, Z, \Pi^{\prime}\right)$. This shows that $P$ is shift-proof, as claimed.

Combining Lemma 4.13 with Theorem 4.4, we get the next corollary.

Corollary 4.14. The pattern 123134 is partial-matching equivalent to the pattern 123413 , and the pattern 123314 is partial-matching equivalent to the pattern 123431.

\section{Bijections involving 'hybrid' matchings}

In this section, we prove the two equivalence relations

$$
123412 \sim 123142 \text { and } 123421 \sim 123241
$$

The two proofs share the same basic structure. In both cases, we construct the bijection between two pattern avoiding classes by composing bijections between classes of 'hybrid' matchings. Informally, a hybrid matching is a matching that avoids two different forbidden patterns in its different parts.

Although the two proofs in this section are similar, we prefer to present them separately. We do not know whether the two results can be generalized to a wider class of forbidden patterns.

\subsection{The patterns 123412 and 123142}

In this subsection, we show that the two patterns 123412 and 123142 are equivalent. Let us write $\sigma=123412$ and $\tau=123142$.

Let $\mu=\mu_{1} \cdots \mu_{2 n}$ be a matching with $n$ edges. For an integer $\ell \in[n]$, we say that $\mu$ contains $\sigma$ at level $\ell$ if $\mu$ contains a subsequence $s=s_{1} s_{2} s_{3} s_{4} s_{5} s_{6}$ order-isomorphic to $\sigma$ such that $s_{2}=s_{6}=\ell$ (in other words, the symbol '2' of $\sigma$ corresponds to the symbol $\ell$ of $s$ ). Similarly, we say that $\mu$ contains $\tau$ at level $\ell$ if $\mu$ has an occurrence $s$ of $\tau$ in which the symbol ' 2 ' of $\tau$ corresponds to the symbol $\ell$ of $s$.

For an integer $\ell \geqslant 1$, a matching $\mu=\mu_{1} \cdots \mu_{2 n}$ is an $\ell$-hybrid if it satisfies the following two conditions:

- For each $\ell^{\prime}<\ell, \mu$ avoids $\sigma$ at level $\ell^{\prime}$.

- For each $\ell^{\prime} \geqslant \ell, \mu$ avoids $\tau$ at level $\ell^{\prime}$.

Notice that 1-hybrid matchings are precisely the matchings that avoid $\tau$, while $(n+1)$ hybrid matchings are precisely the matchings that avoid $\sigma$. Thus, to show that $\sigma$ and $\tau$ are equivalent, it is enough to show that the number of $\ell$-hybrid matchings does not depend on $\ell$. 
Lemma 5.1. For any $\ell \in[n]$, the number of $\ell$-hybrid matchings of order $n$ is equal to the number of $(\ell+1)$-hybrid matchings of order $n$.

Proof. Fix a level $\ell$ and an order $n$. Our goal is to construct a bijection $f$ between $\ell$-hybrid and $(\ell+1)$-hybrid matchings of order $n$.

Let $\mu$ be a matching of order $n$. Express $\mu$ as $\mu=a \ell b \ell c$, where $a, b$ and $c$ are (possibly empty) subwords of $\mu$ delimited by the two occurrences of $\ell$.

Let $x_{1}$ be the element of $\mu$ corresponding the first occurrence of the symbol $\ell+1$, and let $x_{2}$ be the element of $\mu$ corresponding to the second occurrence of $\ell+1$. Let $k \geqslant 0$ be the number of elements of $b$ that are larger than $\ell+1$, and let $y_{1}, y_{2}, \ldots, y_{k}$ be the sequence of all such elements, in the order in which they appear in $b$.

Note that $\mu$ contains $\sigma$ at level $\ell$ if and only if $k>0$ and $b$ contains a symbol smaller than $\ell$ that appears to the right of $y_{1}$. Similarly, $\mu$ contains $\tau$ at level $\ell$ if and only if $k>0$ and $b$ contains a symbol smaller than $\ell$ anywhere between $x_{1}$ and $y_{k}$.

Assume now that $\mu$ is an $\ell$-hybrid matching. We may express $b$ as

$$
b=b^{(0)} x_{1} b^{(1)} y_{1} b^{(2)} y_{2} \cdots y_{k-1} b^{(k)} y_{k} b^{(k+1)},
$$

where $x_{1}, y_{1}, \ldots, y_{k}$ are the elements defined above, and $b^{(0)}, \ldots, b^{(k+1)}$ are possibly empty subwords of $b$. Since $\mu$ is an $\ell$-hybrid, it avoids $\tau$ at level $\ell$, and in particular, none of the subwords $b^{(1)}, \ldots, b^{(k)}$ may contain a symbol smaller than $\ell$. This means that the subwords $b^{(1)}, \ldots, b^{(k)}$ are either empty or consist of a single element $x_{2}$.

Define now the word $b^{\prime}$ obtained from $b$ by exchanging the positions of $b^{(1)}$ and $b^{(k+1)}$. In other words,

$$
b^{\prime}=b^{(0)} x_{1} b^{(k+1)} y_{1} b^{(2)} y_{2} \cdots y_{k-1} b^{(k)} y_{k} b^{(1)} .
$$

Define a new matching $\mu^{\prime}=a \ell b^{\prime} \ell c$. We claim that $\mu^{\prime}$ is an $(\ell+1)$-hybrid matching. It is immediate that $\mu^{\prime}$ is indeed a canonical sequence representing a matching. It is also easy to see that $\mu^{\prime}$ avoids $\sigma$ at level $\ell$.

Let us now check that $\mu^{\prime}$ avoids $\sigma$ at all levels below $\ell$. Suppose this is not the case. Then there are four values $u<v<w<x$ such that $v<\ell$ and $\mu^{\prime}$ has a subsequence $s$ equal to uvwxuv. We may assume without loss of generality that $x=w+1=v+2$, and that the occurrences of $w$ and $x$ in $s$ correspond to the first occurrence of $w$ and $x$ in $\mu^{\prime}$. That means, however, that $s$ contains no element of $\mu^{\prime}$ larger than $\ell$, except possibly the element $x_{1}$. It follows that $s$ is also a subsequence of $\mu$, because the mapping $\mu \mapsto \mu^{\prime}$ only affects the symbols to the right of $x_{1}$, and it does not change the relative positions of two symbols smaller than $\ell$. This is a contradiction.

Let us now check that $\mu^{\prime}$ does not contain $\tau$ at any level greater than $\ell$. We proceed again by contradiction. Assume that there are four values $u<v<w<x$, such that $v>\ell$ and $\mu^{\prime}$ has a subsequence $s=s_{1} s_{2} s_{3} s_{4} s_{5} s_{6}$, whose symbols have values equal to uvwuxv. We may assume that $w=v+1$ and that the symbol $s_{3}$ is the first occurrence of $w$ in $\mu^{\prime}$. If $u>\ell+1$ then $s$ is also a subsequence of $\mu$ because the mapping $\mu \mapsto \mu^{\prime}$ does not change the relative positions of symbols larger than $\ell+1$. We may thus assume that $u \leqslant \ell+1$. 
Let us distinguish several cases, depending on the position of $s_{3}$ and $s_{5}$ in $\mu^{\prime}$. Note that the symbols $s_{3}$ and $s_{5}$ can only appear in $b^{\prime}$ or in $c$. If both these symbols appear in $b^{\prime}$, then $s_{3}=y_{i}$ and $s_{5}=y_{j}$ for some $i<j$. Necessarily, $u \geqslant \ell+1$, because no two symbols $y_{i}, y_{j}$ have a symbol smaller than $\ell+1$ between them. From this, we easily deduce that $s$ is also a subsequence of $\mu$, which is impossible.

If both $s_{3}$ and $s_{5}$ are in $c$, then all the four symbols $s_{3} s_{4} s_{5} s_{6}$ appear in this order in $c$ and hence also in $\mu$. Recall that these symbols have values wuxv. Since the first occurrence of $u$ and $v$ in $\mu$ must precede the first occurrence of $w$, we again conclude that $\mu$ must contain the subsequence uvwuxv.

Lastly, suppose that $s_{3}$ is in $b^{\prime}$ and $s_{5}$ is in $c$. We may then replace the two occurrences of $u$ with the two occurrences of $\ell$ and conclude that both $\mu$ and $\mu^{\prime}$ contain a subsequence $\ell v w \ell x v$ which is order-isomorphic to $\tau$. In all cases, we obtain a contradiction.

Let us define a mapping $f$ by $f(\mu)=\mu^{\prime}$. We have seen that $f$ maps $\ell$-hybrid matchings to $(\ell+1)$-hybrid matchings. It is easy to see that $f$ can be inverted and we routinely check that it maps $(\ell+1)$-hybrid matchings onto $\ell$-hybrid matchings. This shows that $f$ is the required bijection.

Corollary 5.2. The patterns 123412 and 123142 are equivalent.

\subsection{The patterns 123421 and 123241}

Our next goal is to establish the equivalence of the two patterns $\pi=123421$ and $\rho=123241$. Our argument follows the same basic idea as the proof from the previous subsection. Let us adapt the terminology of the previous subsection to the new pair of patterns. We say that a matching $\mu$ contains $\pi$ (or $\rho$ ) at level $\ell$ if $\mu$ has a subsequence $s$ order-isomorphic to $\pi$ in which the symbol $\ell$ corresponds to the symbol ' 2 ' in $\pi$ (or $\rho$ ). We say that a matching $\mu$ is an $\ell$-hybrid if it avoids $\pi$ at all levels $1,2, \ldots, \ell-1$, and it avoids $\rho$ at all levels $\ell, \ell+1, \ldots, n$.

We now show that the number of $\ell$-hybrid matchings of order $n$ does not depend on $\ell$, which means that 123421 and 123241 are equivalent.

Lemma 5.3. For any $\ell \in[n]$, then number of $\ell$-hybrid matchings of order $n$ is equal to the number of $(\ell+1)$-hybrid matchings of order $n$.

Proof. Fix $\ell$ and $n$, with $\ell \leqslant n$. We will describe an involution $f$ on the set of matchings of order $n$, which will act as a bijection between $\ell$-hybrids and $(\ell+1)$-hybrids.

Let $\mu$ be a matching of order $n$. Let us say that a symbol of the sequence $\mu$ is small if its value is smaller than $\ell$, let us say that a symbol is large if its value is larger than $\ell$, and let us say that a large symbol is very large if it is larger than $\ell+1$. Let $\mu_{i}$ be the leftmost large symbol of $\mu$ (which is necessarily equal to $\ell+1$ ), let $\mu_{j}$ be the second occurrence of the symbol $\ell$ in $\mu$, and let $\mu_{k}$ be the rightmost small symbol of $\mu$.

If $i>j$ or $j>k$, then $\mu$ clearly avoids both $\pi$ and $\rho$ at level $\ell$. In such case, we define $f(\mu)=\mu$. Assume now that $i<j<k$. We may decompose the sequence $\mu$ as

$$
\mu=a \mu_{i} b \mu_{j} c \mu_{k} d
$$


where $a, b, c$ and $d$ are (possibly empty) sequences of symbols. Note that $\mu$ contains $\pi$ at level $\ell$ if and only if $b$ has at least one very large symbol, and that $\mu$ contains $\rho$ at level $\ell$ if and only if $c$ has at least one very large symbol. If both $b$ and $c$ contain very large symbols, then $\mu$ contains both $\pi$ and $\rho$ at level $\ell$, while if neither $b$ nor $c$ has a very large symbol, then $\mu$ avoids both $\pi$ and $\rho$ at level $\ell$. In such cases, we define $f(\mu)=\mu$.

Assume now that exactly one of the two words $b$ and $c$ contains a very large symbol. Let $w=w_{1} w_{2} \cdots w_{p}$ denote the sequence $b \ell c$, i.e., $w$ is the subsequence of $\mu$ formed by the symbols of $\mu$ between $\mu_{i}$ and $\mu_{k}$. We now define a new sequence $w^{\prime}=w_{1}^{\prime} w_{2}^{\prime} \cdots w_{p}^{\prime}$ obtained from $w$ by reordering some of its symbols. The sequence $w^{\prime}$ has the property that whenever $w_{i}$ is a small symbol, then $w_{i}^{\prime}=w_{i}$, i.e., the small symbols of $w$ and $w^{\prime}$ coincide. Let $x=x_{1} x_{2} \cdots x_{q}$ be the subsequence of $w$ formed by all its non-small symbols, and let $x^{\prime}=x_{1}^{\prime} x_{2}^{\prime} \cdots x_{q}^{\prime}$ be determined by these rules:

- If the first symbol of $x$ is $\ell$ (i.e., $x_{1}=\ell$ ) then define $x^{\prime}$ by

$$
x^{\prime}=x_{2} x_{3} \ldots x_{q} \ell
$$

- If the last symbol of $x$ is $\ell$, define

$$
x^{\prime}=\ell x_{1} x_{2} \cdots x_{q-1} .
$$

- If the first symbol of $x$ is $\ell+1$ and the second symbol is $\ell$, define

$$
x^{\prime}=x_{3} x_{4} \cdots x_{q} \ell(\ell+1) .
$$

- If the last symbol of $x$ is $\ell+1$, immediately preceded by $\ell$, define

$$
x^{\prime}=(\ell+1) \ell x_{1} x_{2} \cdots x_{q-2} .
$$

The four cases above are mutually exclusive and cover all possibilities. This follows from the assumption that $w$ has at least one very large symbol, and that either all the very large symbols precede $\ell$ or all of them follow $\ell$. Let $w^{\prime}$ be the sequence obtained from $w$ by replacing the subsequence $x$ of $w$ by the subsequence $x^{\prime}$. Note that the very large symbols appear in $w^{\prime}$ in the same order as they appear in $w$.

Let $\mu^{\prime}$ be the sequence of symbols obtained from $\mu$ by replacing the subsequence $w$ with the subsequence $w^{\prime}$, i.e., $\mu^{\prime}=a \mu_{i} w^{\prime} \mu_{k} d$. Since the very large symbols in $w$ appear in the same order as the very large symbols in $w^{\prime}$, we easily see that $\mu^{\prime}$ is a canonical sequence of a matching. We may then define $f(\mu)=\mu^{\prime}$.

Notice that the mapping $f$ defined above is an involution which maps the matchings that avoid $\pi$ at level $\ell$ onto matchings that avoid $\rho$ at level $\ell$.

It is also easy to verify that for every $\ell^{\prime}<\ell, \mu$ avoids $\pi$ at level $\ell^{\prime}$ if and only if $\mu^{\prime}$ avoids $\pi$ at level $\ell^{\prime}$. To see this, notice that if $\mu$ has a subsequence $x \ell^{\prime} y z \ell^{\prime} x$ orderisomorphic to $\pi$, then $\mu$ also has such a subsequence that only uses the small symbols possibly together with the first occurrence of $\ell$ and the first occurrence of $\ell+1$. Thus, at least one occurrence of $\pi$ at level $\ell^{\prime}$ is preserved by $f$. 
Let us now show that for any $\ell^{\prime}>\ell, \mu$ contains $\rho$ at level $\ell^{\prime}$ if and only if $f(\mu)$ contains $\rho$ at level $\ell^{\prime}$. Suppose $\mu$ has a subsequence $s=s_{1} s_{2} s_{3} s_{4} s_{5} s_{6}$ order-isomorphic to $\rho$, with $s_{2}=s_{4}=\ell^{\prime}$. We only need to deal with the case when $\mu \neq f(\mu)$. Recall that $\mu_{k}$ is the rightmost small symbol of $\mu$.

If the whole subsequence $s$ belongs to the prefix $\mu_{1} \mu_{2} \cdots \mu_{k}$ of $\mu$, then we can assume without loss of generality that $s_{6}$ corresponds to $\mu_{k}$. Then the word $w$ contains the subsequence $s_{3} s_{4} s_{5}$. It is easy to check that $w^{\prime}$ must contain this subsequence as well, and from this we easily deduce that $f(\mu)$ has $s$ as a subsequence, so $f(\mu)$ contains $\rho$.

Assume now that the symbol $s_{6}$ of $s$ appears to the right of $\mu_{k}$. Then all the symbols of $s$ are large, and all the symbols of $s$ that appear in $w$ are very large. Since the very large symbols in $w$ form the same subsequence as the very large symbols of $w^{\prime}$, we see that $f(\mu)$ has $s$ as a subsequence.

We conclude that $f$ maps $\ell$-hybrid matchings onto $(\ell+1)$-hybrid matchings, as claimed.

Corollary 5.4. The two partial matchings 123421 and 123241 are equivalent.

\section{Enumerations of short patterns}

We now apply the results of the previous sections to give a summary of equivalence classes among patterns of small size. We also give explicit formulas for the counting functions $\mathfrak{m}_{n}(\tau)$, where available. We then summarize the results in Tables 2-5. In each of the tables, the patterns are ordered by increasing (apparent) growth rate of $\mathfrak{m}_{n}(\tau)$.

Table 2: Number of matchings in $\mathcal{M}_{n}(\tau)$, where $\tau$ is a pattern of length four.

\begin{tabular}{|l|r|ll|}
\hline$\tau$ & $\mathfrak{m}_{7}(\tau)$ & Formula & Reference \\
\hline \hline 1233 & 0 & 0 & Observation 3.1 \\
\hline 1234 & 0 & 0 & Observation 3.1 \\
\hline 1223,1232 & 13 & $2 n-1$ & Lemma 3.4 \\
\hline 1213,1231 & 239 & $(2.1)$ & Corollary 4.12 \\
\hline 1212,1221 & 429 & $\frac{1}{n+1}\left(\begin{array}{c}2 n \\
n\end{array}\right)$ & {$[9]$, Fact 2.3 } \\
\hline 1123 & 1001 & $\frac{3}{2 n+1}\left(\begin{array}{c}2 n+1 \\
n-1\end{array}\right)$ & {$[8]$} \\
\hline 1122 & 5040 & $n !$ & Observation 3.3 \\
\hline
\end{tabular}

\subsection{Patterns of length four}

The results of this paper allow us to provide the full classification of patterns of length four with respect to their equivalence, and to enumerate the corresponding pattern avoiding matchings. The results are listed in Table 2. 


\subsection{Patterns of length five}

For patterns of length five, we are able to classify all the equivalence classes, and give explicit formulas for most, but not all, of the counting functions $\mathfrak{m}_{n}(\tau)$. The results are summarized in Table 3. The formulas for $\mathfrak{m}_{n}(12313)$ and $\mathfrak{m}_{n}(11233)$ are derived in the next two subsections.

Table 3: Number of matchings in $\mathcal{M}_{n}(\tau)$, where $\tau$ is a pattern of length five. For the patterns in bold, we do not have explicit formulas.

\begin{tabular}{|l|r|ll|}
\hline$\tau$ & $\mathfrak{m}_{7}(\tau)$ & Formula & Reference \\
\hline \hline 12344 & 0 & 0 & Observation 3.1 \\
\hline 12345 & 0 & 0 & Observation 3.1 \\
\hline 12334,12343 & 143 & $(2 n-1)(2 n-3)$ & Lemma 3.4 \\
\hline 12324,12342 & 1287 & $(2 n-1) \mathfrak{m}_{n-1}(1213)$ & Lemma 3.4 \\
\hline 12323,12332 & 1716 & $\left(\begin{array}{c}2 n-1 \\
n\end{array}\right)$ & Lemma 3.4 \\
\hline 12314,12341 & 3263 & $(2.2)$ & Corollary 4.12 \\
\hline 12313 & 3804 & $(6.1)$ & \\
\hline 12234 & 3861 & $3\left(\begin{array}{c}2 n-1 \\
n+1\end{array}\right)$ & Lemma 3.4 \\
\hline $\mathbf{1 2 3 3 1}$ & 5787 & & \\
\hline $\mathbf{1 2 1 3 4}$ & 7472 & & [4], Fact 2.6 \\
\hline $12123,12132,12213$, & & & Lemma 3.4 \\
$12231,12312,12321$ & 7752 & $\frac{1}{2 n+1}\left(\begin{array}{c}3 n \\
n\end{array}\right)$ & Lemma 3.10 \\
\hline 12233 & 9360 & $(2 n-1)(n-1) !$ & Lemma 3.5 \\
\hline 11233,12133 & 11743 & $(6.2)$ & \\
\hline $\mathbf{1 1 2 3 4}$ & 16171 & \multicolumn{2}{|c|}{} \\
\hline 11223,11232 & 30869 & $\sum_{j=1}^{n} j !\left(\begin{array}{c}2 n-j-1 \\
j-1\end{array}\right)$ &
\end{tabular}

\subsection{Enumeration of 12313 -avoiding matchings}

Let $\mu$ be a matching of order $n$. Let $e_{1}, \ldots, e_{n}$ be the edges of $\mu$, where each $e_{i}$ connects a pair of vertices $x_{i}<y_{i}$, and the edges are numbered in such a way that $x_{1}<x_{2}<\cdots<x_{n}$. We say that edges $e_{i}$ and $e_{j}$ cross, if $x_{i}<x_{j}<y_{i}<y_{j}$, or $x_{j}<x_{i}<y_{j}<y_{i}$. We say that $e_{j}$ is nested below $e_{i}$ if $x_{i}<x_{j}<y_{j}<y_{i}$. The following observation, whose routine proof we omit, characterizes 12313-avoiding matchings.

Observation 6.1. A matching $\mu$ avoids 12313 if and only for every two edges $e_{i}, e_{j}$ that cross each other, we have either $i=j+1$ or $j=i+1$.

Using this observation, it is not hard to see that the structure of any 12313-avoiding matching $\mu$ is described by exactly one of the following three cases (see Figure 4):

- The edge $e_{1}$ of $\mu$ is not crossed by any other edge. Then the vertices between vertex $x_{1}=1$ and vertex $y_{1}$ induce an arbitrary (possibly empty) 12313-avoiding matching $\sigma$, and the vertices to the right of $y_{1}$ induce an arbitrary (possibly empty) 12313-avoiding matching $\pi$. 
- The edge $e_{1}$ is crossed by $e_{2}$, and $y_{1}=3$. Then $\mu$ begins with the three symbols 121 , and the edges $e_{2}, e_{3}, \ldots, e_{n}$ form an arbitrary 12313 -avoiding matching of order $n-1$.

- The edge $e_{1}$ is crossed by $e_{2}$, and $y_{1}>3$. From this, it follows that $x_{3}<y_{1}$, and since $e_{3}$ cannot cross $e_{1}$, it does not cross $e_{2}$ either. Hence, $e_{2}$ is not crossed by any edge other than $e_{1}$. It follows that the vertices between $x_{2}$ and $y_{1}$ induce a nonempty 12313-avoiding matching $\sigma$, the vertices between $y_{1}$ and $y_{2}$ induce a possibly empty 12313-avoiding matching $\pi$, and the vertices to the right of $y_{2}$ induce a possibly empty 13213-avoiding matching $\tau$.

Furthermore, any matching whose structure corresponds to one of these three cases is clearly 12313-avoiding.
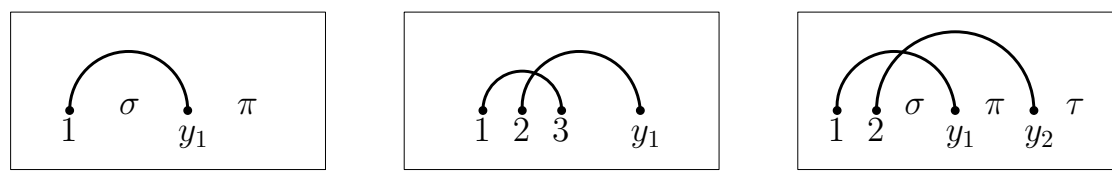

Figure 4: The matchings avoiding 12313.

Let $f(x)$ be the generating function for $\mathfrak{m}_{n}(12313)$. The decompositions of 12313avoiding matching describe above imply the identity

$$
f(x)=1+x f^{2}(x)+x(f(x)-1)+x^{2} f^{2}(x)(f(x)-1),
$$

which is equivalent to

$$
f(x)=1+x f^{2}(x)+\frac{x^{2}}{1-x} f^{3}(x) .
$$

Let $g(x, y)$ be a generating function such that $g(x, y)=x y(g(x, y)+1)^{2}\left(1+\frac{x}{1-x}(g(x, y)+\right.$ $1)$ ). Clearly, $g(x, 1)=f(x)-1$. Using the Lagrange inversion formula (for example, see [15, Theorem 5.1.1]), we get that

$$
\begin{aligned}
{\left[y^{n}\right] g(x, y) } & =\frac{1}{n}\left[a^{n-1}\right]\left(x(a+1)^{2}\left(1+\frac{x}{1-x}(a+1)\right)\right)^{n} \\
& =\sum_{i=0}^{n-1}\left(\begin{array}{c}
2 n \\
i
\end{array}\right)\left(\begin{array}{c}
n \\
n-1-i
\end{array}\right) \frac{x^{2 n-1-i}}{n(1-x)^{n}},
\end{aligned}
$$

Thus,

$$
f(x)=1+\sum_{i \geqslant 1} \sum_{j=0}^{i-1} \frac{x^{2 i-1-j}}{i(1-x)^{i}}\left(\begin{array}{c}
2 i \\
j
\end{array}\right)\left(\begin{array}{c}
i \\
j+1
\end{array}\right),
$$

and hence the number of 12313-avoiding matchings on [2n] is given by

$$
\sum_{i=1}^{n} \sum_{j=0}^{i-1} \frac{1}{i}\left(\begin{array}{c}
2 i \\
j
\end{array}\right)\left(\begin{array}{c}
i \\
j+1
\end{array}\right)\left(\begin{array}{c}
n+j-i \\
i-1
\end{array}\right) .
$$




\subsection{The pattern 11233}

We now derive a formula for $\mathfrak{m}_{n}(11233)$. Let $\mu$ be a matching. Let $e_{i}, x_{i}$ and $y_{i}$ have the same meaning as in the previous subsection. We say that the edge $e_{i}$ of $\mu$ is short, if $y_{i}<x_{n}$. An edge is long if it is not short. In particular, the edge $e_{n}$ is always long.

By definition, if $e_{i}$ is a long edge then $x_{i} \leqslant x_{n}<y_{i}$. It follows that every two long edges are either crossing or nesting.

We may easily check that a matching $\mu$ avoids 11233 if and only if it satisfies the following two conditions.

1. The submatching of $\mu$ induced by the short edges avoids 112 ; in other words, every two short edges are nested. Thus, if $\mu$ has $s$ short edges, then these edges induce a submatching isomorphic to $123 \cdots s s(s-1) \cdots 1$.

2. If $e_{i}$ is a long edge different from $e_{n}$ and $e_{j}$ is a short edge, then $x_{i}<y_{j}$.

We may now easily infer that the number of 11233-avoiding matchings with $\ell$ long edges and $s$ short edges is equal to $\ell !\left(\begin{array}{c}\ell+s-1 \\ s\end{array}\right)$, where the factor $\ell$ ! corresponds to the

number of possible mutual arrangements of the long edges, while $\left(\begin{array}{c}\ell+s-1 \\ s\end{array}\right)$ is the number of possibilities to choose the relative position of the short edges and the left endpoints of the first $\ell-1$ long edges (the left endpoint of the last long edge is always the rightmost left endpoint of the whole matching).

Summing over all possible values of $\ell$, we get the formula

$$
\mathfrak{m}_{n}(11233)=\sum_{\ell=1}^{n} \ell !\left(\begin{array}{l}
n-1 \\
\ell-1
\end{array}\right) .
$$

\subsection{Patterns of length six and seven}

In this subsection, we summarize the equivalence classes for patterns of length six and seven. We verified by computer enumeration that no other patterns of these lengths are equivalent. For patterns of length six, our results confirm all the equivalences suggested by computer enumeration.

On the other hand, there are several patterns of length seven which appear to be equivalent based on computer enumeration, but their equivalence does not follow from our results. These patterns are marked in bold in Table 5. We also list these plausible equivalences explicitly in a conjecture.

Conjecture 6.2. Apart from the equivalences implied by the results of this paper, the partial matchings of length seven also satisfy the following equivalence relations:

- $1234413 \sim 1234431$,

- $1234213 \sim 1231342$, and

- $1233124 \sim 1233142 \sim 1233241$. 
To save space, we only list the nontrivial equivalence classes, and we omit the enumeration data. Complete data, as well as the source code of the program used for the enumerations, are available from the second author's website [16].

The equivalence classes of patterns of length six and seven are listed in Table 4 and Table 5, respectively.

Table 4: Nonsingleton equivalence classes of patterns of length six.

\begin{tabular}{|l|l|}
\hline Pattern & Reference \\
\hline \hline 123445,123454 & Lemma 3.4 \\
\hline 123435,123453 & Lemma 3.4 \\
\hline 123434,123443 & Lemma 3.4 \\
\hline 123425,123452 & Lemma 3.4 \\
\hline 123415,123451 & Corollary 4.12 \\
\hline $123234,123243,123324$, & \\
$123342,123423,123432$ & Lemma 3.4 \\
\hline 122344,123244 & Lemma 3.4 \\
\hline $123134,123143,123413$ & Fact 2.7, Corollary 4.14 \\
\hline 121344,123144 & Lemma 3.10 \\
\hline $123314,123341,123431$ & Fact 2.5, Corollary 4.14 \\
\hline $123124,123142,123214$, & Corollaries $4.12,5.2$ and 5.4, \\
$123241,123412,123421$ & Fact 2.7 \\
\hline 123132,123213 & Corollary 4.3 \\
\hline $123123,123312,123321$ & Fact 2.1 \\
\hline 121332,122313 & Observation 3.8 \\
\hline 121234,122134 & Fact 2.3 \\
\hline 122334,122343 & Lemma 3.4 \\
\hline $112334,112343,121334,121343$ & Lemmas 3.7 and 3.10, Fact 2.4 \\
\hline $112323,112332,121233,122133$ & Lemmas 3.5 and 3.9 \\
\hline 112324,112342 & Lemma 3.5 \\
\hline
\end{tabular}

Table 5: Nonsingleton equivalence classes of patterns of length seven.

\begin{tabular}{|l|l|}
\hline Pattern & Reference \\
\hline \hline 1234556,1234565 & Lemma 3.4 \\
\hline 1234545,1234554 & Lemma 3.4 \\
\hline 1234546,1234564 & Lemma 3.4 \\
\hline 1234536,1234563 & Lemma 3.4 \\
\hline 1233455,1234355 & Lemma 3.4 \\
\hline 1234526,1234562 & Lemma 3.4 \\
\hline $1234345,1234354,1234435$, & \\
$1234453,1234534,1234543$ & Lemma 3.4 \\
\hline 1232455,1234255 & Lemma 3.4 \\
\hline 1234516,1234561 & Corollary 4.12 \\
\hline $1234245,1234254,1234524$ & Lemma 3.4 \\
\hline 1231455,1234155 & Lemma 3.10 \\
\hline $1234425,1234452,1234542$ & Lemma 3.4 \\
\hline & continued on next page
\end{tabular}


Table 5: Nonsingleton equivalence classes of patterns of length seven.

\begin{tabular}{|l|l|}
\hline Pattern & Reference \\
\hline \hline $1234235,1234253,1234325$, & \\
$1234352,1234523,1234532$ & Lemma 3.4 \\
\hline 1234451,1234541 & Fact 2.5 \\
\hline 1234243,1234324 & Lemma 3.4 \\
\hline $1234234,1234423,1234432$ & Lemma 3.4 \\
\hline 1232443,1233424 & Lemma 3.4 \\
\hline $1234125,1234215,1234512,1234521$ & Corollary 4.12 \\
\hline 1233445,1233454 & Lemma 3.4 \\
\hline$(\mathbf{1 2 3 4 4 1 3}),(\mathbf{1 2 3 4 4 3 1 )}$ & \\
\hline $1223434,1223443,1232344,1233244$ & Lemma 3.4 \\
\hline 1232345,1233245 & Lemma 3.4 \\
\hline $1223445,1223454,1232445,1232454$ & Lemma 3.4 \\
\hline $1123434,1123443,1213443,1213434$ & Lemmas 3.7 and 3.10 \\
\hline 1234412,1234421 & Fact 2.1 \\
\hline $1234132,1234213,(\mathbf{1 2 3 1 3 4 2})$ & Corollary 4.3 \\
\hline $1213445,1213454,1231445,1231454$ & Lemmas 3.7 and 3.10 \\
\hline $1212344,1213244,1221344$, & Fact 2.3, \\
$1223144,1231244,1232144$ & Lemma 3.10 \\
\hline 1123344,1213344 & Lemma 3.10 \\
\hline$(\mathbf{1 2 3 3 1 2 4}),(\mathbf{1 2 3 3 1 4 2}),(\mathbf{1 2 3 3 2 4 1})$ & \\
\hline $1231234,1233214,1233412$, & Facts 2.1 and 2.5, \\
\hline $1233421,1234123,1234312,1234321$ & Corollary 4.12 \\
\hline 1231245,1232145 & Fact 2.3 \\
\hline 1123445,1123454 & Fact 2.4 \\
\hline 1223435,1223453 & Lemma 3.4 \\
\hline 1122344,1123244 & Corollary 3.6 \\
\hline $1123435,1123453,1213435,1213453$ & Lemmas 3.7 and 3.10 \\
\hline $1123234,1123243,1123324$, & \\
$1123342,1123423,1123432$ & Lemma 3.5 \\
\hline 1212345,1221345 & Fact 2.3 \\
\hline 1123345,1213345 & Lemma 3.5 \\
\hline 1123425,1123452 & Lemma 3.5 \\
\hline $1212334,1212343,1221334,1221343$ & Facts 2.2 and 2.4 \\
\hline 1122334,1122343 & Fact 2.4 \\
\hline & \\
\hline
\end{tabular}

\section{References}

[1] J. Backelin, J. West, G. Xin: Wilf equivalence for singleton classes, Advances in Applied Mathematics 38 (2007) 133-148.

[2] W.Y.C. Chen, E.Y.P. Deng, R.R.X. Du, R.P. Stanley, C.H.F. Yan: Crossings and nestings of matchings and partitions, Trans. Amer. Math. Soc. 359 (2007) 15551575.

[3] W.Y.C. Chen, E.Y.P. Deng, R.R.X. Du: Reduction of $m$-regular noncrossing partitions, Europ. J. Combin. 26 (2005), No. 2, 237-243. 
[4] W.Y.C. Chen, T. Mansour, and S.H.F. Yan: Matchings avoiding partial patterns, Elect. J. Combin. 13 (2006) \#R112.

[5] W.Y.C. Chen, G. Xin, and T.Y.J. Zhang: Enumeration of $k$-stack sortable matchings and partitions, Permutation Patterns 2007, Cambridge University Press to appear.

[6] A. de Mier: $k$-noncrossing and $k$-nonnesting graphs and fillings of Ferrers diagrams, Combinatorica 27(6) (2007) 699-720.

[7] V. Jelínek, T. Mansour: On pattern-avoiding partitions, Elect. J. Combin. 15(1) (2008) \#R39.

[8] V. Jelínek, N.Y. Li, T. Mansour, and S.H.F. Yan: Matchings avoiding partial patterns and lattice paths, Elect. J. Combin. 13 (2006) \#R89.

[9] M. Klazar: On abab-free and abba-free set partitions, Europ. J. Combin. 17 (1996) $53-68$.

[10] C. Krattenthaler: Growth diagrams, and increasing and decreasing chains in fillings of Ferrers shapes, Adv. Appl. Math. 37:3 (2006) 404-431.

[11] M. Rubey: Increasing and decreasing sequences in fillings of moon polyominoes, arXiv:math/0604140v5.

[12] B. Sagan: Pattern avoidance in set partitions, Ars Combinatoria 94 (2010) 79-96.

[13] Z. Stankova and J. West: A new class of Wilf-equivalent permutations, J. Alg. Combin. 15 (2002) 271-290.

[14] R.P. Stanley: Catalan Addendum, http://www-math.mit.edu/ rstan/ec/catadd.pdf.

[15] H. Wilf, Generatingfunctionology, (1990), Academic Press, New York.

[16] http://math.haifa.ac.il/toufik/enumerative/match_table6.html 